\title{
HOMOLOGICAL DIMENSION AND CRITICAL EXPONENT OF KLEINIAN GROUPS
}

\author{
MichaEL KAPOVICH
}

\begin{abstract}
We prove an inequality between the relative homological dimension of a Kleinian group $\Gamma \subset \operatorname{Isom}\left(\mathbb{H}^{n}\right)$ and its critical exponent. As an application of this result we show that for a geometrically finite Kleinian group $\Gamma$, if the topological dimension of the limit set of $\Gamma$ equals its Hausdorff dimension, then the limit set is a round sphere.
\end{abstract}

\section{Introduction}

One of the frequent themes in the theory of Kleinian groups is establishing a relation between the abstract algebraic properties of a Kleinian group and its geometric properties, determined by its action on the hyperbolic space. Ahlfors finiteness theorem and Mostow rigidity theorem are among the most important examples of such a relation. In this paper we establish a relation between two invariants of a Kleinian group: virtual homological dimension (an algebraic invariant) and the critical exponent (a geometric invariant). We refer the reader to section 2 for the precise definitions.

Given a Kleinian group $\Gamma \subset \operatorname{Isom}\left(\mathbb{H}^{n}\right)$, consider the set $\mathcal{P}$ of its maximal virtually abelian subgroups of virtual rank $\geq 2$, i.e. the elements of $\mathcal{P}$ are maximal subgroups which contain a subgroup isomorphic to $\mathbb{Z}^{2}$. Form the maximal subset

$$
\Pi:=\left\{\Pi_{i}, i \in I\right\} \subset \mathcal{P}
$$

of pairwise nonconjugate elements of $\mathcal{P}$. In other words, $\Pi$ consists of representatives of cusps of rank $\geq 2$ in $\Gamma$.

We let $\operatorname{vhd}_{R}(\Gamma, \Pi)$ and $\operatorname{vcd}_{R}(\Gamma, \Pi)$ denote the virtual homological and cohomological dimension of $\Gamma$ relative to $\Pi$, where $R$ is a commutative ring with a unit. (Instead of working with virtual dimensions, one can use the (co)homological dimension with respect to fields of zero characteristic,

Keywords and phrases: Kleinian groups, homological dimension, Hausdorff dimension, critical exponent

AMS Mathematics Subject Classification: 30F40, 20F67 
or, more generally, rings where the order of every finite subgroup of $\Gamma$ is invertible.) Let $\delta(\Gamma)$ be the critical exponent of $\Gamma$.

Our main result is

Theorem 1.1. For every virtually torsion-free Kleinian group $\Gamma$ we have

$$
\operatorname{vhd}_{R}(\Gamma, \Pi)-1 \leq \delta(\Gamma) .
$$

Corollary 1.2. Suppose that the pair $(\Gamma, \Pi)$ has finite type, e.g. $\Gamma$ admits a finite $K(\Gamma, 1)$ and the set $\Pi$ is finite. Then

$$
\operatorname{cd}_{R}(\Gamma, \Pi)-1 \leq \delta(\Gamma) .
$$

One, therefore, can regard these results as either nontrivial lower bounds on the critical exponent, or as vanishing theorems for relative (co)homology groups of $\Gamma$ with arbitrary twisted coefficients. These results also can be viewed as generalizing the classical inequality

$$
\operatorname{dim}(Z) \leq \operatorname{dim}_{H}(Z)
$$

for compact metric spaces $Z$, see [HuW]. Here $\operatorname{dim}(Z)$ is the topological dimension and $\operatorname{dim}_{H}(Z)$ is the Hausdorff dimension.

As an application of Corollary 1.2 we prove

Theorem 1.3. Suppose that $\Gamma \subset \operatorname{Isom}\left(\mathbb{H}^{n}\right)$ is a nonelementary geometrically finite group so that the Hausdorff dimension of its limit set equals its topological dimension $d$. Then the limit set of $\Gamma$ is a round $d$-sphere, i.e. $\Gamma$ preserves a $d+1$-dimensional subspace $H \subset \mathbb{H}^{n}$ and $H / \Gamma$ has finite volume.

This theorem was first proved by Rufus Bowen [Bowe] for convexcocompact quasi-fuchsian subgroups of Isom $\left(\mathbb{H}^{3}\right)$. Bowen's theorem was extended by Bishop and Jones [BisJ] to subgroups of $\operatorname{Isom}\left(\mathbb{H}^{3}\right)$ with parabolic elements. Bowen's result was generalized by Chenbo Yue $[\mathrm{Y}]$ to convexcocompact subgroups of $\operatorname{Isom}\left(\mathbb{H}^{n}\right)$ whose limit sets are topological spheres, although his argument did not need the latter assumption. Note that the arguments of Yue do not work in the presence of parabolic elements. For cocompact discrete groups of isometries of $C A T(-1)$ spaces, an analogue of Theorem 1.3 was proved by Bonk and Kleiner [BoK], see also the work of Besson, Gallot and Courtois [BCG2]. The latter paper was the inspiration for our work. X. Xie $[\mathrm{X}]$ proved a special case of Theorem 1.3 under the assumption that $\Gamma$ is isomorphic to the fundamental group of a (possibly noncompact) complete hyperbolic $d+1$-dimensional manifold of finite volume.

Conjecture 1.4. Suppose that $\Gamma$ is a finitely generated Kleinian group in $\operatorname{Isom}\left(\mathbb{H}^{n}\right)$. Then, 
1. $d=\operatorname{vcd}_{R}(\Gamma, \Pi)-1 \leq \delta(\Gamma)$.

2. In the case of equality, $\Gamma$ is geometrically finite and its the limit set is a round $d$-sphere in $S^{n-1}$.

Another application of our main theorem is the following property of groups with small critical exponent:

Corollary 1.5. Suppose that $\delta(\Gamma)<1$ and $\Gamma$ is of type $F P_{2}$, e.g. is finitely presented. Then $\Gamma$ is virtually free.

Problem 1.6 (Cf. Theorem 1.3 in $[$ BisJ] and $[\mathrm{H}]$.). Is it true that every finitely generated Kleinian group $\Gamma$ with $\delta(\Gamma)<1$ is geometrically finite? Is it true that such group is a classical Schottky-type group?

The proofs of our results are generalizations of the proofs due to Besson, Courtois and Gallot in [BCG2]. Our main contribution in comparison to their paper is treatment of arbitrary coefficient modules, working with relative homology groups and handling manifolds whose injectivity radius is not bounded from below. The most nontrivial technical ingredient of our paper is the existence of the natural maps introduced in [BCG2] and their properties established in that paper.

In the case of a Kleinian subgroup $\Gamma \subset \operatorname{Isom}\left(\mathbb{H}^{3}\right)$, our main theorem easily follows from the well-known facts about $\Gamma$. It suffices to consider the case when $\Gamma$ is finitely generated and torsion free. If $\delta(\Gamma)=2$, then Theorem 1.1 states that

$$
\operatorname{vhd}(\Gamma, \Pi) \leq 3 .
$$

The latter inequality immediately follows from the fact that the hyperbolic manifold $\mathbb{H}^{3} / \Gamma$ is a 3-dimensional Eilenberg-MacLane space for $\Gamma$. Assume therefore that $\delta(\Gamma)<2$. Then it follows from the solution of the tameness conjecture $[\mathrm{A}],[\mathrm{CG}]$ (which, in turn, implies Ahlfors' measure zero conjecture) and [BisJ], that $\Gamma$ is geometrically finite. Therefore either $\Gamma$ is a Schottky-type group or it contains a finitely generated quasi-fuchsian subgroup $\Phi \subset \Gamma$, whose limit set is a topological circle. In the latter case,

$$
2 \geq \operatorname{vhd}_{R}(\Gamma, \Pi) \geq \operatorname{vhd}_{R}(\Phi, \Pi \cap \Phi)=2,
$$

while

$$
\delta(\Gamma) \geq \delta(\Phi) \geq 1 .
$$

This implies the inequality

$$
1=2-1=\operatorname{vhd}_{R}(\Gamma, \Pi)-1 \leq 1 \leq \delta(\Gamma) .
$$

If $\Gamma$ is a Schottky-type group, then

$$
\Gamma \cong F_{k} * \Pi_{1} * \cdots * \Pi_{m},
$$

where $\Pi_{i} \in \Pi$ for $i=1, \ldots, m$. Therefore $\operatorname{vhd}_{R}(\Gamma, \Pi)=1$ and Theorem 1.1 trivially follows. 
Sketch of the proof of Theorem 1.1. Let $\epsilon$ be a positive number which is smaller than the Margulis constant $\mu_{n}$ for $\mathbb{H}^{n}$. Let $\delta:=\delta(\Gamma)$. We assume that $\Gamma$ is torsion free. We sketch the proof under the following assumption:

There exists a thick triangulation of the hyperbolic manifold $M=\mathbb{H}^{n} / \Gamma$, i.e. a triangulation $T$ and a number $L<\infty$, so that every $i$-simplex in $T$ not contained in the $\epsilon$-thin part $M_{(0, \epsilon]}$ of $M$ is $L$-bilipschitz diffeomorphic to the standard Euclidean $i$-simplex. (Existence of such triangulation was recently proven by Bill Breslin [Br]. Breslin's proof follows the line of arguments sketched by Thurston in the proof of [T1, Prop. 5.11.2].)

Suppose that $\operatorname{hd}_{R}(\Gamma, \Pi)>\delta+1$. Then for some $q>\delta+1$, there exists a flat bundle $\mathbb{V}$ over the manifold $M$, so that

$$
H_{q}\left(M, M_{(0, \epsilon]} ; \mathbb{V}\right) \neq 0 .
$$

Pick a chain $\zeta \in C_{q}(M ; \mathbb{V})$ which projects to a nonzero class $[\zeta]$ in $H_{q}\left(M, M_{(0, \epsilon]} ; \mathbb{V}\right)$. We then extend $\zeta$ to the $\epsilon$-thin part of $M$, to a locally finite absolute cycle $\hat{\zeta}$ of finite volume. Besson, Courtois and Gallot in [BCG2] proved existence of a natural map $F: M \rightarrow M$ which is (properly) homotopic to the identity and satisfies

$$
\operatorname{vol}\left(F_{\#}(\hat{\zeta})\right) \leq\left(\frac{\delta+1}{q}\right)^{q} \operatorname{vol}(\hat{\zeta})
$$

Since $q>\delta+1$, the locally finite cycle $\hat{\zeta}_{k}:=F_{\#}^{k}(\hat{\zeta})$ satisfies

$$
\lim _{k \rightarrow \infty} \operatorname{vol}\left(\hat{\zeta}_{k}\right)=0 \text {. }
$$

Then we use the deformation lemma of Federer and Fleming to deform (for large $k$ ) the cycle $\hat{\zeta}_{k}$ to a locally finite cycle $\hat{\xi}_{k}$ which is supported in the $q-1$-skeleton of $T$ away from $M_{(0, \epsilon]}$. Therefore $\hat{\xi}_{k}$ determines zero homology class in $H_{q}\left(M, M_{(0, \epsilon]} ; \mathbb{V}\right)$. Since $F^{k}$ is properly homotopic to the identity (with uniform control on the length of the tracks of the homotopy) we conclude that $[\zeta]$ is trivial as well, which is a contradiction.

Instead of relying upon Breslin's result, we use instead a map $\eta$ from $M$ to a simplicial complex $X$, which is the nerve of an appropriate cover of $M$. The map $\eta$ is $L_{\kappa}$-Lipschitz on the $\kappa$-thick part of $M$ for every $\kappa>0$. This allows us to do the deformation arguments in $X$ rather than in $T$. This line of arguments is borrowed from [G, $\S 5.32]$.

Acknowledgements. This work was partially supported by the NSF grant DMS 0405180. Most of this paper was written when the author was visiting the Max Plank Institute for Mathematics in Bonn. I am grateful to Gérard Besson and Gilles Courtois for sharing with me an early version of [BCG2], to Leonid Potyagailo for motivating discussions and to 
Xiangdong Xie for telling me about his preprint [X]. I am thankful to the referee for useful suggestions and comments.

\section{Preliminaries}

\subsection{Geometric preliminaries.}

Basics of Kleinian groups. We let $\mathbb{H}^{n}$ denote the hyperbolic $n$ space, $S^{n-1}$ the ideal boundary of $\mathbb{H}^{n}$, and $\operatorname{Isom}\left(\mathbb{H}^{n}\right)$ the isometry group of $\mathbb{H}^{n}$. A Kleinian group is a discrete isometry group of $\mathbb{H}^{n}$. The limit set of a Kleinian group $\Gamma$ is denoted $\Lambda(\Gamma)$. A Kleinian group $\Gamma$ is called elementary if its limit set contains at most 2 points. A Kleinian group is elementary if and only if it is virtually abelian. We let

$$
\operatorname{Hull}(\Lambda(\Gamma)) \subset \mathbb{H}^{n}
$$

denote the convex hull of $\Lambda(\Gamma)$ in $\mathbb{H}^{n}$.

Let $\Gamma \subset \operatorname{Isom}\left(\mathbb{H}^{n}\right)$ be a Kleinian group, $x \in \mathbb{H}^{n}$ be a point and $\epsilon$ be a positive real number. Let

$$
\Gamma_{x, \epsilon} \subset \Gamma
$$

denote the subgroup generated by the elements $\gamma \in \Gamma$ such that

$$
d(x, \gamma(x)) \leq \epsilon .
$$

Then, according to Kazhdan-Margulis lemma, for every $n$ there is a constant $\mu_{n}>0$, called the Margulis constant, such that $\Gamma_{x, \mu_{n}}$ is elementary, for every Kleinian subgroup $\Gamma \subset \operatorname{Isom}\left(\mathbb{H}^{n}\right)$ and every point $x \in \mathbb{H}^{n}$.

Thick-thin decomposition of hyperbolic manifolds. For a point $x$ in a Riemannian manifold $M$ (possibly with convex boundary) define

$$
\operatorname{InRad}_{M}(x)
$$

to be the injectivity radius of $M$ at $x$. Then the function $\operatorname{InRad}_{M}$ is 1Lipschitz, i.e. it satisfies

$$
\left|\operatorname{InRad}_{M}(x)-\operatorname{InRad}_{M}\left(x^{\prime}\right)\right| \leq d\left(x, x^{\prime}\right) .
$$

Suppose that $M$ is a metrically complete connected hyperbolic manifold with convex boundary. Let $\tilde{M}$ denote the universal cover of $M$. For $0<$ $\epsilon<\mu_{n}$ consider the thick-thin decomposition

$$
M=M_{(0, \epsilon]} \cup M_{[\epsilon, \infty)} .
$$

Here thin part $K=M_{(0, \epsilon]}$ of $M$ is the closure of the set of points $x \in M$, such that there exists a homotopically nontrivial loop $\gamma_{x}$ based at $x$, whose length is $<\epsilon$.

Let $K_{i}, i \in J \subset \mathbb{N}$, denote the connected components of $K$.

Lemma 2.1. Each $K_{i}$ is covered by a contractible submanifold $\tilde{K}_{i}$ in $\mathbb{H}^{n}$. 
Proof. We identify $\pi_{1}\left(K_{i}\right)$ with an elementary subgroup $\Pi_{i} \subset \Gamma$. Then $\tilde{K}_{i}=\tilde{K}_{i}(\epsilon)$ is the union

where

$$
\tilde{K}_{i}(\epsilon)=\bigcup_{\gamma \in \Pi_{i} \backslash\{1\}} \tilde{K}_{\epsilon}(\gamma)
$$

$$
\tilde{K}_{\epsilon}(\gamma)=\{z \in \tilde{M}: d(z, \gamma(z)) \leq \epsilon\} .
$$

Each $\tilde{K}_{\epsilon}(\gamma)$ is convex, since the displacement function of $\gamma$ is convex. Of course, the union of convex sets need not be convex and $K_{i}$ is, in general, not convex. We first consider the case when $\Pi_{i}$ is a cyclic hyperbolic subgroup. Let $A=A_{i}$ denote the common axis of the nontrivial elements of $\Pi_{i}$. Then $A$ is contained in each $\tilde{K}_{\epsilon}(\gamma)$. It follows that $\tilde{K}_{i}:=\tilde{K}_{i}(\epsilon)$ is star like with respect to every point of $A$. Therefore $\tilde{K}_{i}$ is contractible.

If $\Pi_{i}$ is parabolic, this argument of course does not apply. Let $\xi=\xi_{i}$ denote the fixed point of $\Pi_{i}$. Then $\tilde{K}_{i}$ is star like with respect to $\xi$. Therefore, every map $f: S^{k} \rightarrow \tilde{K}_{i}(\epsilon)$ can be homotoped to a map $f_{\kappa}: S^{k} \rightarrow \tilde{K}_{i}(\kappa)$ along the geodesics asymptotic to $\xi$, where $\kappa$ and

$$
d(\kappa):=\operatorname{diam}\left(f_{\kappa}\left(S^{k}\right)\right)
$$

can be chosen arbitrarily small. Then $f_{\kappa}\left(S^{k}\right)$ bounds a ball $f_{\kappa}\left(B^{k+1}\right)$ within $d(\kappa)$ from the image of $f_{\kappa}$. Thus

$$
f_{\kappa}\left(B^{k+1}\right) \subset \tilde{K}_{i}(\kappa+2 d(\kappa)) \text {. }
$$

By choosing $\kappa$ so that $\kappa+2 d(\kappa)<\epsilon$, we conclude that $\pi_{k}\left(\tilde{K}_{i}\right)=0$ for all $k$.

Therefore each $K_{i}=K\left(\Pi_{i}, 1\right)$ is an Eilenberg-MacLane space for its fundamental group $\Pi_{i}$.

Critical exponent of a Kleinian group. Let $\Gamma \subset \operatorname{Isom}\left(\mathbb{H}^{n}\right)$ be a Kleinian group. Consider the Poincaré series

$$
f_{s}=\sum_{\gamma \in \Gamma} e^{-s d(\gamma(o), o)}
$$

where $o \in \mathbb{H}^{n}$ is a base-point and $d$ is the hyperbolic metric on $\mathbb{H}^{n}$. Then the critical exponent of $\Gamma$ is

$$
\delta(\Gamma)=\inf \left\{s: f_{s}<\infty\right\} .
$$

Critical exponent has several alternative descriptions. Define

$$
N(R):=\#\{x \in \Gamma \cdot o: d(x, o) \leq R\} .
$$

Then $\delta(\Gamma)$ is the rate of exponential growth of $N(R)$, i.e.

$$
\delta(\Gamma)=\limsup _{R \rightarrow \infty} \frac{\log (N(R))}{R},
$$

see $[\mathrm{N}]$. Lastly, the critical exponent can be interpreted in terms of the geometry of the limit set of $\Gamma$ : 
Theorem 2.2 (See [BisJ], [N], [Su], [Tu].). For every nonelementary Kleinian group $\Gamma \subset \operatorname{Isom}\left(\mathbb{H}^{n}\right)$, we have

1.

$$
\delta(\Gamma)=\operatorname{dim}_{H}\left(\Lambda_{c}(\Gamma)\right) .
$$

In particular, if $\Gamma$ is geometrically finite, $\Lambda(\Gamma) \backslash \Lambda_{c}(\Gamma)$ is at most countable and we obtain

$$
\delta_{\Gamma}=\operatorname{dim}_{H}\left(\Lambda_{c}(\Gamma)\right) .
$$

2. If $\Gamma$ is geometrically finite then either $\Lambda(\Gamma)=S^{n-1}$ or $\delta(\Gamma)<n-1$.

Here $\operatorname{dim}_{H}$ is the Hausdorff dimension and $\Lambda_{c}(\Gamma) \subset S^{n-1}$ is the conical limit set of $\Gamma$.

Thus the critical exponent of a Kleinian group is easy to estimate from above:

$$
\delta(\Gamma) \leq n-1 .
$$

Estimates from below, however, are nontrivial; our main theorem provides such a lower bound.

2.2 Algebraic preliminaries. In this section we collect various definitions and results of homological algebra. We refer the reader to $[\mathrm{Bi}],[\mathrm{BiE}]$ and [Bro] for the detailed discussion. For the rest of the paper, we let $R$ be a commutative ring with a unit denoted 1 . We note that although $[\mathrm{BiE}]$ and [Bro] restrict their discussion to $R=\mathbb{Z}$, the definitions and facts that we will need directly generalize to the general commutative rings.

Suggestion to the reader. For most of the paper, the reader uncomfortable with homological algebra can think of (co)homology of $\Gamma$ with trivial coefficients and of existence of a finite $K(\Gamma, 1)$ instead of the finite type condition for $\Gamma$. However, in the proofs of Theorem 1.3 and Corollary 1.5, we need (co)homology with twisted coefficients as well as the general notion of finite type.

A group $\Gamma$ is said to be of finite type, or $F P$ (over $R$ ), if there exists a resolution by finitely generated projective $R \Gamma$-modules

$$
0 \rightarrow P_{k} \rightarrow P_{k-1} \rightarrow \cdots \rightarrow P_{0} \rightarrow R \rightarrow 0 .
$$

For instance, if there exists a finite cell complex $K=K(\Gamma, 1)$, then $\Gamma$ has finite type for every ring $R$. Every group of finite type is finitely generated, although it does not have to be finitely presented, see [BeB].

More generally, a group $\Gamma$ is said to be of type $F P_{k}$ (over $R$ ), if there exists a partial resolution by finitely generated projective $R \Gamma$-modules

$$
P_{k} \rightarrow P_{k-1} \rightarrow \cdots \rightarrow P_{0} \rightarrow R \rightarrow 0 .
$$


A group $\Gamma$ is said to have cohomological dimension $k$ if $k$ is the least integer such that there exists a resolution by projective $R \Gamma$-modules

$$
0 \rightarrow P_{k} \rightarrow P_{k-1} \rightarrow \cdots \rightarrow P_{0} \rightarrow R \rightarrow 0 .
$$

Lemma 2.3. Suppose that $\Gamma$ is of type $F P_{k}$ and $\operatorname{cd}(\Gamma) \leq k$. Then $\Gamma$ is of type FP.

Proof. See discussion following the proof of Proposition 6.1 in [Bro, Ch. VIII].

A group $\Gamma$ is said to have homological (or weak) dimension $k$ over $R$, if $k$ is the least integer such that there exists a resolution by flat $R \Gamma$-modules

$$
0 \rightarrow F_{k} \rightarrow F_{k-1} \rightarrow \cdots \rightarrow F_{0} \rightarrow R \rightarrow 0 .
$$

Thus the (co)homological dimension of $\Gamma$ equals the (projective) flat dimension of the $\Gamma$-module $R \Gamma$. The cohomological and homological dimensions of $\Gamma$ are denoted by $\operatorname{cd}_{R}(\Gamma)$ and $\operatorname{hd}_{R}(\Gamma)$ respectively. One can restate the definition of (co)homological dimension in terms of vanishing of (co)homologies of $\Gamma$ :

Theorem 2.4 (See [Bi].).

$$
\begin{aligned}
& \operatorname{cd}_{R}(\Gamma)=\sup \left\{n: \exists \text { an } R \Gamma \text {-module } V \text { so that } H^{n}(\Gamma ; V) \neq 0\right\}, \\
& \operatorname{hd}_{R}(\Gamma)=\sup \left\{n: \exists \text { an } R \Gamma \text {-module } V \text { so that } H_{n}(\Gamma ; V) \neq 0\right\} .
\end{aligned}
$$

Theorem 2.5. Let $\Gamma$ be a torsion-free group such that $\operatorname{cd}_{R}(\Gamma) \leq 1$. Then $\Gamma$ is free.

This theorem was originally proven by Stallings $[\mathrm{S}]$ for finitely generated groups and $R=\mathbb{Z}$; his proof was extended by Swan $[\mathrm{Sw}]$ to arbitrary groups. Finally, Dunwoody [D] proved this theorem for arbitrary rings.

REMARK 2.6. One can weaken the torsion-free assumption, by restricting to groups with torsion of bounded order, see [D].

We will need a generalization of these definitions to the relative case. In what follows we let $\Gamma$ be a group and $\Pi$ be a nonempty collection of subgroups

$$
\Pi:=\left\{\Pi_{i}, i \in I\right\} .
$$

Given an $R \Gamma$-module $V$, one defines the relative (co)homology groups

$$
H_{*}(\Gamma, \Pi ; V), \quad H^{*}(\Gamma, \Pi ; V) .
$$

Instead of the algebraic definition of (co)homologies with coefficients in an $R \Gamma$-module $V$, we will be using the topological interpretation, following [BiE, $\S 1.5]$. Let $K:=K(\Gamma, 1)$ be an Eilenberg-MacLane space for $\Gamma$. Let 
$C_{i}:=K\left(\Pi_{i}, 1\right), i \in I$. We assume that the complexes $C_{i}$ are embedded in $K$, so that $C_{i} \cap C_{j}=\emptyset$ for $i \neq j$. Set

$$
C:=\bigcup_{i \in I} C_{i}
$$

Then we will be computing the (co)homologies of the pair $(\Gamma, \Pi)$ using the relative (co)homologies of $(K, C)$. Namely, let $X$ denote the universal cover of $K$. Let $V_{R}$ be the module $V$, regarded as an $R$-module. We obtain the trivial (product) sheaf $\tilde{\mathbb{V}}$ over $X$ with fibers $V_{R}$. We will think of this sheaf as the sheaf of local (horizontal) sections of the product bundle $\mathbb{E}:=X \times V_{R} \rightarrow X$. By abusing the notation we will identify bundles and sheaves of their sections. The group $\Gamma$ acts on this sheaf diagonally:

$$
\gamma \cdot(x, v)=(\gamma(x), \gamma \cdot v), \quad \gamma \in \Gamma .
$$

The bundle $\mathbb{E}$ (and the sheaf $\tilde{\mathbb{V}}$ ) project to the space $K$, to a bundle $\mathbb{V} \rightarrow K$ and its sheaf $\mathbb{V}$ of local horizontal sections. Then we have natural isomorphisms

$$
H^{*}(\Gamma, \Pi ; V) \cong H^{*}(K, C ; \mathbb{V}), \quad H_{*}(\Gamma, \Pi ; V) \cong H_{*}(K, C ; \mathbb{V}) .
$$

We will mostly work with the relative homology groups $H_{*}(K, C ; \mathbb{V})$, which we will think of as the (relative) singular homology of $K$ (rel. $C$ ) with coefficients in $\mathbb{V}$. We refer the reader to $[\mathrm{JM}]$ for the precise definition.

The most important example (for us) of this computation of relative homologies will be when $\Gamma$ is a Kleinian group, the complex $K$ is the hyperbolic manifold $M=\mathbb{H}^{n} / \Gamma$, and the subcomplex $C$ is a disjoint union of Margulis tubes and cusps in $M$. More generally, we will consider the case when $K$ is a metrically complete connected hyperbolic manifold with convex boundary.

We now return to the general case of group pairs $(\Gamma, \Pi)$.

Definition 2.7. The relative (co)homological dimension of $\Gamma$ (rel. $\Pi$ ) is defined as

$$
\begin{aligned}
& \operatorname{cd}_{R}(\Gamma, \Pi)=\sup \left\{n: \exists \text { an } R \Gamma \text {-module } V \text { so that } H^{n}(\Gamma, \Pi ; V) \neq 0\right\}, \\
& \operatorname{hd}_{R}(\Gamma, \Pi)=\sup \left\{n: \exists \text { an } R \Gamma \text {-module } V \text { so that } H_{n}(\Gamma, \Pi ; V) \neq 0\right\} .
\end{aligned}
$$

In the case of $R=\mathbb{Z}$, we will omit the subscript from the notation for the (co)homological dimension. We note that for every $\Gamma$ of finite (relative) homological dimension there exists a finitely generated subgroup $\Gamma^{\prime} \subset \Gamma$ so that

cf. Corollary 4.10 in [Bi].

$$
\operatorname{hd}_{R}(\Gamma, \Pi)=\operatorname{hd}_{R}\left(\Gamma^{\prime}, \Pi \cap \Gamma^{\prime}\right),
$$

Set

$$
R \Gamma / \Pi:=\oplus_{i \in I} R \Gamma / \Pi_{i} .
$$


We have the augmentation $\epsilon: R \Gamma / \Pi \rightarrow R$, given by $\epsilon\left(g \Pi_{i}\right):=1$ for all cosets $g \Pi_{i}$ and all $i$. Following [BiE, §1.1], we set

Then (see $[\mathrm{BiE}, \S 1.1])$

$$
\Delta:=\Delta_{\Gamma / \Pi}:=\operatorname{Ker}(\epsilon) .
$$

$$
\begin{gathered}
H^{k}(\Gamma, \Pi ; V) \cong H^{k-1}(\Gamma ; \operatorname{Hom}(\Delta, V)), \\
H_{k}(\Gamma, \Pi ; V) \cong H_{k-1}(\Gamma ; \Delta \otimes V) .
\end{gathered}
$$

The cohomological and homological dimensions of $(\Gamma, \Pi)$ can be interpreted as flat and projective dimensions of $\Delta=\Delta_{\Gamma / \Pi}$ respectively:

$$
\operatorname{hd}_{R}(\Gamma, \Pi)-1=\text { flat } \operatorname{dim}(\Delta), \quad \operatorname{cd}_{R}(\Gamma, \Pi)-1=\operatorname{proj} \operatorname{dim}(\Delta),
$$

see [BiE, §4.1]. For most of the paper this interpretation of (co)homological dimension will be unnecessary; the only exceptions are Lemmata 2.8 and 2.9 below:

LEMMA 2.8.

$$
\operatorname{hd}_{R}(\Gamma, \Pi) \leq \operatorname{cd}_{R}(\Gamma, \Pi) \leq \operatorname{hd}_{R}(\Gamma, \Pi)+1 .
$$

Proof. The absolute case was proved in [Bi]; the relative case follows from the same arguments as in Bieri's book using the equation (2).

A pair $(\Gamma, \Pi)$ is said to have finite type (over $R$ ) if

1. $\Gamma$ and each $\Pi_{i}$ has type $F P$.

2. The set $I$ is finite.

This condition is stronger than the one considered in [BiE, §4.1]. However it will suffice for our purposes as we are interested in the case where each $\Pi_{i}$ is a finitely generated virtually abelian group. Such groups $\Pi_{i}$ necessarily have finite type.

Note that there is a free finitely generated Kleinian group $\Gamma \subset \operatorname{Isom}\left(\mathbb{H}^{4}\right)$, so that $\Gamma$ contains infinitely many $\Gamma$-conjugacy classes of maximal parabolic subgroups, $[\mathrm{KP}]$. It is unknown if every Kleinian group $\Gamma$ of finite type contains only finitely many conjugacy classes of maximal parabolic subgroups of $\operatorname{rank} \geq 2$.

If $\Gamma \subset \operatorname{Isom}\left(\mathbb{H}^{n}\right)$ is a geometrically finite Kleinian group, then it contains only finitely many conjugacy classes of maximal parabolic subgroups, see [Bow]. Moreover, $\Gamma$ has finite type since its admits a finite $K(\Gamma, 1)$, which is the complement to cusps in the convex core of $\mathbb{H}^{n} / \Gamma$. Therefore in this case $(\Gamma, \Pi)$ has finite type.

Lemma 2.9. If $(\Gamma, \Pi)$ is of finite type, then

1. $\operatorname{cd}_{R}(\Gamma, \Pi)=\operatorname{hd}_{R}(\Gamma, \Pi)$.

2. $\operatorname{cd}_{R}(\Gamma, \Pi)=\sup \left\{n: H^{n}(\Gamma, \Pi ; R \Gamma) \neq 0\right\}$. 
Proof. This theorem was proved in [Bi] (see also [Bro, Ch. VIII, Prop. 6.7]) in the case when $\Pi=\emptyset$. The same arguments go through in the relative case.

Suppose that $\Gamma$ is virtually torsion free, i.e. it contains a finite-index subgroup $\Gamma^{\prime} \subset \Gamma$ which is torsion free. Let $\Pi^{\prime}$ denote the collection of subgroups of $\Gamma^{\prime}$ obtained by intersecting $\Gamma^{\prime}$ with the elements of $\Pi$. One defines the virtual relative (co)homological dimension of $\Gamma$ as

$$
\begin{aligned}
\operatorname{vcd}_{R}(\Gamma, \Pi) & =\operatorname{cd}_{R}\left(\Gamma^{\prime}, \Pi^{\prime}\right), \\
\operatorname{vhd}_{R}(\Gamma, \Pi) & =\operatorname{hd}_{R}\left(\Gamma^{\prime}, \Pi^{\prime}\right) .
\end{aligned}
$$

One can easily show that vcd and $h c d$ are independent of the choice of $\Gamma^{\prime}$ : The absolute case immediately follows from [Bi, Cor. 5.10], the relative case is completely analogous.

Recall that every finitely generated Kleinian group is virtually torsion free by Selberg's lemma; hence it has finite (co)homological dimension.

\section{Volumes of Relative Cycles}

Let $X$ be either a simplicial complex or a Riemannian manifold, possibly with convex boundary. In the case when $X$ is a simplicial complex, we metrize $X$ by identifying each $i$-simplex in $X$ with the standard Euclidean $i$-simplex in $\mathbb{R}^{i+1}$. Let $Y \subset X$ be either a subcomplex or a closed submanifold with piecewise-smooth boundary. Let $\hat{\omega}_{q}$ be the $q$-volume form on $X$ induced by piecewise-Euclidean or Riemannian metric on $X$. Let $\chi$ be the characteristic function of $X \backslash Y$; we define the relative $q$-volume form $\omega_{q}$ by

$$
\omega_{q}:=\chi \cdot \hat{\omega}_{q} .
$$

Let $\mathbb{W} \rightarrow X$ be a flat bundle whose fibers are copies of an $R$-module $V_{R}$. We define the relative volume $\operatorname{Vol}(\zeta, Y)$ for piecewise-smooth singular $q$-chains $\zeta$ in $C_{q}(X, \mathbb{W})$ as follows. Consider first the case when $\zeta=w \otimes \sigma$, where $\sigma: \Delta^{q} \rightarrow X$ is a singular $q$-simplex and $w$ is a (horizontal) section of $\mathbb{W}$ over the support of $\sigma$. Then set

$$
\operatorname{Vol}(\zeta, Y)=\int_{\Delta^{q}} \sigma^{*}\left(\omega_{q}\right)
$$

For a general chain

$$
\zeta=\sum_{i=1}^{s} w_{i} \otimes \sigma_{i}
$$

set

$$
\operatorname{Vol}(\zeta, Y):=\sum_{i=1}^{s} \operatorname{Vol}\left(w_{i} \otimes \sigma_{i}\right)
$$


We set $\operatorname{Vol}(\zeta):=\operatorname{Vol}(\zeta, \emptyset)$. Clearly, the relative volume descends to a function on $Z_{q}(X, Y ; \mathbb{W})$. For a relative homology class $\xi \in H_{q}(X, Y ; \mathbb{W})$, we define the relative volume by

$$
\operatorname{Vol}(\xi, Y):=\inf \{\operatorname{Vol}(\zeta, Y): \xi=[\zeta]\} .
$$

Note that our definition of relative volume does not take the coefficients into account. Suppose that $R$ is a normed ring with a norm $|\cdot|$ and $V$ is a normed $R \Gamma$-module, i.e. it admits a norm $|\cdot|$ such that

$$
|r \gamma \cdot v|=|r| \cdot|v|, \quad \forall r \in R, \gamma \in \Gamma
$$

where $|r|$ is the norm of $r \in R$. For instance, take $V=\mathbb{R} \Gamma$ or $V=\mathbb{R}$, the trivial $\mathbb{R} \Gamma$-module. If $R$ is a normed ring, then the normed modules suffice for calculation of the cohomological dimension of $\Gamma$ over $R$, see [Bro, Ch. VIII, Prop. 2.3].

Then one can define another volume function, which is sensitive to the coefficients:

$$
\operatorname{vol}(w \otimes \sigma, Y)=\int_{\Delta^{q}}|w| \sigma^{*}\left(\omega_{q}\right)
$$

However, as the rings discussed in this paper are general (for instance, we allow finite rings $R$ ), we cannot use this definition.

Problem 3.1. Is it true that for every group $\Gamma$,

$$
\operatorname{cd}_{\mathbb{R}}(\Gamma)=\sup \left\{q: \exists \text { a Banach } \mathbb{R} \Gamma \text {-module } V \text { so that } H^{q}(\Gamma, V) \neq 0\right\} ?
$$

Here a Banach $\mathbb{R} \Gamma$-module is a normed $\mathbb{R} \Gamma$-module which is complete as a normed vector space. Note that the answer is unclear even for groups $\Gamma$ of finite type, since $\mathbb{R} \Gamma$ is not a Banach space.

\section{Coning Off Singular Chains}

Let $M$ be a metrically complete hyperbolic $n$-manifold with convex boundary and $\mathbb{V} \rightarrow M$ be a flat bundle whose fibers are isomorphic to the $R$ module $V_{R}$. Pick $0<\epsilon \leq \mu_{n}$. For a singular chain

we define its $\epsilon$-excision

$$
\sigma \in C_{q}(M ; \mathbb{V}) \text {, }
$$

$$
\operatorname{Exc}_{\epsilon}(\sigma)=\sigma \cap M_{[\epsilon, \infty)} \in C_{q}\left(M_{[\epsilon, \infty)} ; \mathbb{V}\right)
$$

by excising the open submanifold $M_{(0, \epsilon)} \subset M$. The main goal of this section is to define and examine a converse to this procedure.

Let

$$
M_{(0, \epsilon]}=P \cup Q=P_{1} \cup \cdots \cup P_{s} \cup Q_{1} \cup \cdots \cup Q_{l},
$$

where $Q$ is the union of compact components (tubes) $Q_{i}$ of $M_{(0, \epsilon]}$ and $P$ is the union of noncompact components (cusps) $P_{j}$. 
4.1 Extension to the tubes. Suppose that $K=Q_{i} \subset M_{(0, \epsilon]}$ is a component which retracts to a closed geodesic $c \subset K$. Given a singular simplex

$$
\sigma: \Delta^{q} \rightarrow K
$$

we define the extension $\operatorname{ext}(\sigma)$ of $\sigma$ to $\Delta^{q} \times[0,1]$ as follows. For $x \in \Delta^{q}$, $t \in[0,1]$, let $x^{\prime}:=\sigma(x)$ and $x^{\prime \prime} \in c$ be the point nearest to $x^{\prime}$. Choose the point

on the geodesic segment $\overline{x^{\prime} x^{\prime \prime}}$, so that

$$
a=\operatorname{ext} \sigma(x, t)
$$

$$
d(\sigma(x), a)=t d\left(x^{\prime}, x^{\prime \prime}\right) .
$$

We triangulate $\Delta^{q} \times[0,1]$ so that $\operatorname{ext}(\sigma)$ is a singular chain. Finally, extend linearly the operator ext to the entire $C_{*}(K ; \mathbb{V})$.

Suppose that

$$
\zeta \in C_{q+1}(M ; \mathbb{V})
$$

is a chain which projects to a relative cycle in $Z_{q+1}\left(M, M_{(0, \epsilon]} ; \mathbb{V}\right)$. For each tube $Q_{i}$ we consider the extension

$$
\zeta_{i}^{\prime}:=\operatorname{ext}_{Q_{i}}\left(\partial \zeta \cap Q_{i}\right) .
$$

Since each $Q_{i}$ retracts to a closed geodesic contained in $Q_{i}$, we obtain Lemma 4.1. For each $q \geq 1$, the extension

$$
\zeta^{\prime}:=\zeta+\sum_{i=1}^{l} \zeta_{i}^{\prime}
$$

projects to a relative cycle in $Z_{q+1}(M, Q ; \mathbb{V})$.

4.2 Extension to the cusps. Recall that $P \subset M$ is the union of cusps. Given a chain

$$
\zeta \in C_{q+1}(M ; \mathbb{V})
$$

which projects to a relative cycle in

$$
Z_{q+1}(M, P ; \mathbb{V})
$$

we will define a locally finite absolute cycle $\hat{\zeta}$, which is an extension of $\zeta$ to the cusps.

Let $\Delta^{m}$ be the standard $m$-simplex $\left[e_{0}, \ldots, e_{m}\right]$ and $\Delta^{m-1}$ be its face $\left[e_{1}, \ldots, e_{m}\right]$. We parameterize the punctured simplex

$$
\Delta_{\circ}^{m}:=\Delta^{m} \backslash\left\{e_{0}\right\}
$$

as follows. Given a point $z \in \Delta_{\circ}^{m}$, consider the line segment $\overline{e_{0} x} \subset \Delta^{m}$ containing $z$, where $x \in \Delta^{m-1}$. Then

$$
z=t x+(1-t) e_{0}, \quad 0 \leq t \leq 1 .
$$

Therefore we give the point $z$ the coordinates $(x, t), x \in \Delta^{m-1}, t \in[0,1]$. 
Fix a point $\xi \in \partial \mathbb{H}^{n}$ and consider a piecewise-smooth singular simplex

$$
\sigma: \Delta^{m-1} \rightarrow \mathbb{H}^{n}
$$

We define the extension

$$
\operatorname{ext}_{\xi}(\sigma): \Delta_{\circ}^{m} \rightarrow \mathbb{H}^{n}
$$

of $\sigma$ as follows. For the point $y=\sigma(x)$ consider the geodesic ray

$$
\rho=\rho_{y, \xi}:[0, \infty) \rightarrow \mathbb{H}^{n}
$$

emanating from $y$ and asymptotic to $\xi$. We parameterize $\rho$ with the unit speed and set

$$
\operatorname{ext}_{\xi}(\sigma)(x, t):=\rho(-\log (t)) .
$$

Then $\operatorname{ext}_{\xi}(\sigma)$ is a piecewise-smooth proper map. Given a singular chain which is a linear combination

$$
\sigma:=\sum_{i} w_{i} \otimes \sigma_{i}, \quad w_{i} \in V_{R},
$$

we set

$$
\operatorname{ext}_{\xi}(\sigma):=\sum_{i} w_{i} \otimes \operatorname{ext}_{\xi}\left(\sigma_{i}\right) .
$$

This extension satisfies

$$
\partial \operatorname{ext}_{\xi}(\sigma)=\operatorname{ext}_{\xi}(\partial \sigma) .
$$

The extension is invariant under the action of $\operatorname{Isom}\left(\mathbb{H}^{n}\right)$ in the sense that

For a chain

$$
\gamma_{*}\left(\operatorname{ext}_{\xi}(\sigma)\right)=\operatorname{ext}_{\gamma(\xi)}\left(\gamma_{*}(\sigma)\right), \quad \forall \gamma \in \operatorname{Isom}\left(\mathbb{H}^{n}\right) .
$$

$$
\sigma=\sum_{i} w_{i} \otimes \sigma_{i}
$$

we define volume of the "punctured" chain $\operatorname{ext}_{\xi}(\sigma)$ by

$$
\operatorname{Vol}\left(\operatorname{ext}_{\xi}(\sigma)\right):=\sum_{i} \operatorname{Vol}\left(\operatorname{ext}_{\xi}\left(\sigma_{i}\right)\right) .
$$

Lemma 4.2. For every $q$-chain $\sigma, q \geq 1$, we have

$$
\operatorname{Vol}\left(\operatorname{ext}_{\xi}(\sigma)\right) \leq q \cdot \operatorname{Vol}(\sigma) .
$$

Proof. It suffices to prove the inequality in the case of a singular simplex $\sigma$. We will work in the upper half-space model of $\mathbb{H}^{n}$, so that $\xi=\infty$. By subdividing the chain $\sigma$ appropriately we can assume that $\sigma(x)=(x, f(x))$, is the graph of a continuous map

$$
f: \Omega \rightarrow(0, \infty),
$$

where $\Omega$ is a bounded domain in $\mathbb{R}^{q} \subset \mathbb{R}^{n-1} \subset \partial \mathbb{H}^{n}$, and $f$ is smooth on the interior of $\Omega$. Then

$$
\operatorname{Vol}(\sigma)=\int_{\Omega} \frac{\sqrt{1+|\nabla f|^{2}}}{f(x)^{q}} d x \geq \int_{\Omega} \frac{d x}{f(x)^{q}}
$$


and

Therefore

$$
\operatorname{Vol}\left(\operatorname{ext}_{\xi}(\sigma)\right)=\int_{\Omega} \int_{f(x)}^{\infty} \frac{d t}{t^{q+1}} d x=\int_{\Omega} \frac{q d x}{f(x)^{q}}
$$

$$
\operatorname{Vol}\left(\operatorname{ext}_{\xi}(\sigma)\right) \leq q \cdot \operatorname{Vol}(\sigma) .
$$

Suppose now that $\zeta \in C_{q+1}(M ; \mathbb{V})$,

$$
\partial \zeta=\sum_{j=1}^{s} \zeta_{j}, \quad \zeta_{j} \in C_{q}\left(P_{j} ; \mathbb{V}\right), i=1, \ldots, s .
$$

For every singular chain

$$
\sigma=\zeta_{j}=\sum_{i} w_{i} \otimes \sigma_{i} \in C_{q}\left(P_{j}, \mathbb{V}\right),
$$

we define a locally finite singular chain

as follows. Lift each $\sigma_{i}$ to a chain

$$
\operatorname{ext}(\sigma) \in C_{q+1}^{l f}\left(P_{j}, \mathbb{V}\right)
$$

$$
\tilde{\sigma}=\sum_{i} v_{i} \otimes \tilde{\sigma}_{i} \in C_{q}\left(\tilde{P}_{j}, V_{R}\right), v_{i} \in V_{R}
$$

where $\tilde{P}_{j} \subset \mathbb{H}^{n}$ is a component of the preimage of $P_{j}$; let $\Pi_{j}$ be the stabilizer of $\tilde{P}_{j}$ in $\Gamma$. Let $\xi=\xi_{j}$ be a point fixed by $\Pi_{j}$.

REMARK 4.3. Our construction does not depend on whether $\Pi_{j}$ is parabolic or hyperbolic. In parabolic case we, of course, have unique fixed point.

If $\Pi_{j}$ is hyperbolic, then the extension chain $\operatorname{ext}(\sigma)$ below is not going to be locally finite in $M$, as it "spins towards" a closed geodesic.

Now extend $\tilde{\sigma}$ to a punctured chain $\operatorname{ext}_{\xi}(\tilde{\sigma})$. Finally, project the latter to a punctured chain $\operatorname{ext}(\sigma)$ via the universal cover $\mathbb{H}^{n} \rightarrow M$. Triangulate $\Delta_{\circ}^{q+1}$, so that $\operatorname{ext}(\sigma)$ is a locally-finite singular chain. Since $\tilde{P}_{j}$ is star like with respect to $\xi$, it follows that

$$
\operatorname{ext}(\sigma) \in C_{q+1}^{l f}\left(P_{j}, \mathbb{V}\right) \text {. }
$$

Invariance of the extension $\operatorname{ext}_{\xi}$ under $\operatorname{Isom}\left(\mathbb{H}^{n}\right)$, ensures that $\operatorname{ext}(\sigma)$ does not depend on the choice of the lifts $\tilde{\sigma}_{i}$. We also have

$$
\partial \operatorname{ext}(\sigma)=\operatorname{ext}(\partial \sigma) \text {. }
$$

Lemma 4.2 implies

Corollary 4.4 (Cf. [T2].). $\operatorname{Vol}(\operatorname{ext}(\sigma)) \leq q \cdot \operatorname{Vol}(\sigma)$.

Lastly, set

$$
\hat{\zeta}:=\zeta+\sum_{j=1}^{s} \operatorname{ext}\left(\zeta_{j}\right)
$$


The excision operation obviously extends to the locally-finite chains $\operatorname{ext}(\sigma)$ and we obtain

$$
\operatorname{Exc}_{\kappa}(\hat{\zeta}) \in Z_{q+1}\left(M, M_{(0, \kappa])} ; \mathbb{V}\right)
$$

for every $0<\kappa \leq \epsilon$. It is clear that

$$
\left[\operatorname{Exc}_{\kappa}(\hat{\zeta})\right]=[\hat{\zeta}] \in H_{q}\left(M, M_{(0, \epsilon]} ; \mathbb{V}\right) \text {. }
$$

Therefore we obtain

Proposition 4.5. Let $0<\epsilon \leq \mu_{n}$, and $P \subset M_{(0, \epsilon]}$ be the union of cusps. Then for every chain $\zeta \in C_{q+1}(M ; \mathbb{V})$, which projects to a relative homology class in $H_{q}(M, P ; \mathbb{V})$, there exists a locally finite cycle

so that

$$
\hat{\zeta} \in Z_{q+1}^{l f}(M ; \mathbb{V})
$$

1. $\operatorname{Vol}(\hat{\zeta}) \leq q \operatorname{Vol}(\partial \zeta)+\operatorname{Vol}(\zeta)$

2. $\left[\operatorname{Exc}_{\kappa}(\hat{\bar{\zeta}})\right]=[\hat{\zeta}] \in H_{q}\left(M, M_{(0, \epsilon]} ; \mathbb{V}\right)$ for every $0<\kappa \leq \epsilon$.

\section{Cuspidal Homology}

Let $M$ be a metrically complete hyperbolic $n$-manifold with convex boundary. Let $\pi_{1}(M) \cong \Gamma \subset \operatorname{Isom}\left(\mathbb{H}^{n}\right)$ and $\Pi$ be the collection of cusps in $\Gamma$, i.e. $\Pi$ consists of representatives of $\Gamma$-conjugacy classes of maximal parabolic subgroups of $\Gamma$. Note that here we allow parabolic subgroups of rank 1. Let $\mathbb{V}$ be a flat bundle over $M$ associated with an $R \Gamma$-module $V$. The elements $\Pi_{i}$ of $\Pi$ correspond to the components $P_{i}$ of $P \subset M_{\left(0, \mu_{n}\right]}$. Given $0<\epsilon \leq \mu_{n}$, consider the thick-thin decomposition

and let

$$
M=M_{(0, \epsilon]} \cup M_{[\epsilon, \infty)}
$$

$$
P_{\epsilon}:=P \cap M_{(0, \epsilon]} .
$$

We then have the direct system

$$
\left(M, P_{\epsilon}\right) \rightarrow\left(M, P_{\epsilon^{\prime}}\right), \quad 0<\epsilon \leq \epsilon^{\prime} \leq \mu_{n} .
$$

These maps induce isomorphisms

$$
H_{*}\left(M, P_{\epsilon} ; \mathbb{V}\right) \rightarrow H_{*}\left(M, P_{\epsilon^{\prime}} ; \mathbb{V}\right) .
$$

We therefore identify

$$
\lim _{\epsilon} H_{*}\left(M, P_{\epsilon} ; \mathbb{V}\right) \cong H_{*}\left(M, P_{\mu_{n}} ; \mathbb{V}\right) \cong H^{*}(\Gamma, \Pi ; V) .
$$

We will refer to this direct limit as the cuspidal homology of $M$,

$$
H_{*}^{\text {cusp }}(M ; \mathbb{V}) .
$$

We have an obvious homomorphism

$$
\operatorname{Exc}: H_{*}^{l f}(M ; \mathbb{V}) \rightarrow H_{*}^{c u s p}(M ; \mathbb{V})
$$


given by the excision. The advantage of working with the above direct limit is the following:

Suppose that $f: M \rightarrow M$ is a proper $L$-Lipschitz map. Then $f$ induces a self-map

$$
f:\left\{\left(M, P_{\epsilon}\right)\right\} \rightarrow\left\{\left(M, P_{\epsilon}\right)\right\}
$$

of the direct system; the latter clearly induces an isomorphism

$$
f_{*}: H_{*}^{\text {cusp }}(M ; \mathbb{V}) \rightarrow H_{*}^{\text {cusp }}(M ; \mathbb{V}) .
$$

If $f$ induces the identity automorphism of $\Gamma$, then $f_{*}=I d$.

\section{Partition of Unity and a Map to the Nerve for a Hyperbolic $\boldsymbol{n}$-Manifold}

Fix a number $\epsilon>0$. Suppose that $M$ is a complete hyperbolic $n$-manifold. (In this section we do not allow $M$ to have boundary.) Given a covering $\mathcal{U}$ of $M$ by contractible open sets with contractible intersections, $M$ is homotopy-equivalent to the nerve of $\mathcal{U}$. The goal of this section is to get a homotopy-equivalence with controlled Lipschitz constant. The Lipschitz constant will be bounded on the thick part of $M$. This construction is standard (cf. [G, §5.32]), we include it for the sake of completeness.

Our first goal is to find an appropriate covering $\mathcal{U}$ by convex metric balls $B_{\epsilon_{j}}\left(x_{j}\right)$ in $M$, whose radii $\epsilon_{j}$ are multiples of the injectivity radii of $x_{j}$.

Choose $0<\alpha<1$ and define the function

$$
\tau(x):=\alpha \min \left(\operatorname{InRad}_{M}(x), \frac{\epsilon}{2}\right) .
$$

Note that $\tau$ is a continuous function which is constant on $M_{[\epsilon, \infty)}$. Recall that $B_{r}(x)$ denotes the open $r$-ball centered at $x$.

Lemma 6.1. Suppose that $x, y \in M$ are such that

Then

$$
B_{\tau(x)}(x) \cap B_{\tau(y)}(y) \neq \emptyset .
$$

$$
\frac{\tau(x)}{\tau(y)} \leq \frac{1+\alpha}{1-\alpha} .
$$

Proof. If $x, y \in M_{[\epsilon, \infty)}$ then $\tau(x)=\tau(y)=\alpha \epsilon / 2$ and we are done. We consider the case

$$
\tau(x) \geq \tau(y)=\alpha \cdot \operatorname{InRad}_{M}(y) .
$$

Then the inequality (1) implies that

$$
\alpha^{-1}(\tau(x)-\tau(y)) \leq \operatorname{InRad}_{M}(x)-\operatorname{InRad}_{M}(y) \leq d(x, y) \leq \tau(x)+\tau(y) .
$$

Therefore

$$
\frac{\tau(x)}{\tau(y)} \leq \frac{1+\alpha}{1-\alpha} .
$$


Lemma 6.2. Let $\beta>0$ be such that

$$
0<\frac{\beta}{\frac{1}{2}-\beta}<\frac{1-\alpha}{1+\alpha} .
$$

Then there exists a covering $\mathcal{D}$ of the manifold $M$ by the open balls

$$
D_{i}:=B_{\tau\left(x_{i}\right) / 2}\left(x_{i}\right), \quad i \in I,
$$

so that

$$
B_{\beta \tau\left(x_{j}\right)}\left(x_{j}\right) \cap B_{\beta \tau\left(x_{i}\right)}\left(x_{i}\right)=\emptyset, \quad \forall x_{i} \neq x_{j} .
$$

Proof. We construct the set $E$ of centers $x_{i}, i \in I$, of the above balls as follows. Choose a maximal set

$$
E=\left\{x_{i}, i \in I\right\} \subset M,
$$

satisfying (3). Suppose that $\mathcal{D}:=\left\{D_{j}, j \in I\right\}$ is not a covering of $M$. Then there exists

$$
x \in M \text { so that } d\left(x, x_{i}\right) \geq \tau\left(x_{i}\right) / 2, \quad \forall i \in I .
$$

Suppose that there exists $i \in I$ so that

$$
B_{\beta \tau(x)}(x) \cap B_{\beta \tau\left(x_{i}\right)}\left(x_{i}\right) \neq \emptyset .
$$

Then

$$
\frac{\tau(y)}{2} \leq d(x, y)<\beta(\tau(x)+\tau(y))
$$

for $y:=x_{i}$. By Lemma 6.1,

$$
\frac{\tau(x)}{\tau(y)} \leq \frac{1+\alpha}{1-\alpha}
$$

and, by combining these inequalities, we get

$$
\frac{\beta}{\frac{1}{2}-\beta} \geq \frac{1-\alpha}{1+\alpha} \text {. }
$$

This contradicts out choice of $\beta$. Therefore

$$
B_{\beta \tau(x)}(x) \cap B_{\beta \tau\left(x_{i}\right)}=\emptyset, \quad \forall i \in I,
$$

which contradicts maximality of $E$. Hence

is the required covering of $M$.

$$
\mathcal{D}=\left\{B_{\tau\left(x_{i}\right) / 2}\left(x_{i}\right), x_{i} \in E\right\}
$$

We leave it to the reader to verify that if $0<\alpha \leq 1 / 8,0<\beta$, and

then

$$
0<\frac{\beta}{\frac{1}{2}-\beta}<\frac{1-\alpha}{1+\alpha},
$$

$$
1+\beta \leq \frac{1-\alpha}{\alpha(1+\alpha)} .
$$

We let $\operatorname{vol}(r)$ denote the volume of a hyperbolic $r$-ball in $\mathbb{H}^{n}$. Let $\omega_{n}$ denote the volume of the Euclidean $n$-ball of the unit radius. Then

$$
\operatorname{vol}(r) \geq \omega_{n} r^{n} \text {. }
$$


Moreover, whenever $r \leq 1$, we have

$$
\operatorname{vol}(r) \leq \omega_{n} \cdot 2^{n-1} r^{n}
$$

Given a choice of $\alpha$ and $\beta$ as above, define the covering

$$
\mathcal{U}:=\left\{B_{i}=B_{\tau\left(x_{i}\right)}\left(x_{i}\right): i \in I\right\}
$$

of the manifold $M$, where $E=\left\{x_{i}, i \in I\right\}$ is as in Lemma 6.2.

Lemma 6.3. Assume that $0<\alpha \leq 1 / 8,0<\beta$ and

$$
0<\frac{\beta}{\frac{1}{2}-\beta}<\frac{1-\alpha}{1+\alpha} .
$$

Then the covering $\mathcal{U}$ has multiplicity at most

$$
\frac{2^{2 n-1}}{\beta^{n}} \text {. }
$$

Proof. Suppose that

$$
y \in \bigcap_{j \in J} B_{j}
$$

for some $J \subset I$ of cardinality $m$. Let $\nu:=\max \left\{\tau\left(x_{j}\right), j \in J\right\}$. Then for every $j \in J$,

$$
B_{\beta \tau\left(x_{j}\right)}\left(x_{j}\right) \subset B_{(1+\beta) \nu}(y) .
$$

The balls $B_{\beta \tau\left(x_{j}\right)}\left(x_{j}\right), j \in J$, are pairwise disjoint by the definition of $\mathcal{U}$. Therefore

$$
\sum_{j \in J} \operatorname{Vol}\left(B_{\beta \tau\left(x_{j}\right)}\left(x_{j}\right)\right) \leq \operatorname{Vol}\left(B_{(1+\beta) \nu}(y)\right) .
$$

Recall that

$$
\frac{\tau(y)}{\alpha} \leq \operatorname{InRad}_{M}(y) .
$$

By combining this with the inequality

$$
\nu=\tau\left(x_{j}\right) \leq \frac{1+\alpha}{1-\alpha} \tau(y)
$$

(for some $j \in J$ ) and the inequality (4), we obtain

$$
(1+\beta) \nu \leq \operatorname{InRad}_{M}(y) .
$$

Therefore the ball $B_{(1+\beta) \nu}(y)$ is contained in a normal ball and thus has the volume

$$
\operatorname{vol}((1+\beta) \nu) .
$$

By Lemma 6.1,

$$
\tau\left(x_{i}\right) \geq \nu \frac{1-\alpha}{1+\alpha}, \quad \forall i \in J .
$$

Hence

$m \cdot \operatorname{vol}\left(\beta \nu \frac{1-\alpha}{1+\alpha}\right) \leq \sum_{i \in J} \operatorname{Vol}\left(B_{\beta \tau\left(x_{i}\right)}\left(x_{i}\right)\right) \leq \operatorname{Vol}\left(B_{(1+\beta) \nu}(y)\right)=\operatorname{vol}((1+\beta) \nu)$. 
Combining this with the inequalities (5) and (6), we obtain

$$
m \leq 2^{n-1}\left[\frac{(1+\beta)(1+\alpha)}{\beta(1-\alpha)}\right]^{n} .
$$

Since $\alpha \leq 1 / 8$ and $\beta<1 / 2$, it follows that

$$
m \leq \frac{2^{2 n-1}}{\beta^{n}} .
$$

We now fix $\alpha>0$ and $\beta>0$ such that

and

$$
\alpha \leq \frac{1}{8}
$$

$$
0<\frac{\beta}{\frac{1}{2}-\beta}<\frac{1-\alpha}{1+\alpha} .
$$

For instance, take $\alpha=\beta=1 / 8$. We then obtain

Proposition 6.4. There exists a function $m(n, \epsilon): \mathbb{N} \times(0, \infty) \rightarrow \mathbb{N}$, with the following property. For every complete hyperbolic $n$-manifold $M$, there exits a countable subset $E=\left\{x_{i}, i \in I\right\} \subset M$ and a collection of positive numbers $\left\{\rho_{i}, i \in I\right\}$, so that

1. Set $\mathcal{D}:=\left\{D_{i}=B_{\rho_{i} / 2}\left(x_{i}\right): x_{i} \in E\right\}$ and $\mathcal{U}:=\left\{B_{i}=B_{\rho_{i}}\left(x_{i}\right): x_{i} \in E\right\}$. Then $\mathcal{D}$ (and therefore $\mathcal{U}$ ) covers $M$.

2. For every $x_{i} \in M_{[\epsilon, \infty)}$,

3. For every $x_{i} \in M_{(0, \epsilon)}$,

$$
\rho_{i}=\frac{\epsilon}{16} .
$$

$$
\frac{\operatorname{InRad}_{M}\left(x_{i}\right)}{8}=\rho_{i} \leq \frac{\epsilon}{16} .
$$

4. The multiplicity of the covering $\mathcal{U}$ is at most $m(n, \epsilon)$.

Proof. Set $\rho_{i}:=\tau\left(x_{i}\right)$. The rest follows from Lemmata 6.2 and 6.3.

Corollary 6.5. Suppose that

$$
x \in \bigcap_{j \in J} B_{j}
$$

where $J \subset I$. Then part 3 of Proposition 6.4 in conjunction with the inequality (1), implies that the union

$$
\bigcup_{j \in J} B_{j}
$$

is contained in a normal neighborhood $N_{x}$ of $x$.

We now associate a partition of unity $\left\{\eta_{i}, i \in I\right\}$ to the covering $\mathcal{U}$ as in Proposition 6.4. For the metric $r$-ball $B_{r}(o) \subset \mathbb{H}^{n}$ we define a bumpfunction $b_{r}(x)$ on $\mathbb{H}^{n}$ supported in $B_{r}(o)$, so that 
1. $0 \leq b_{r}(x) \leq 1, \forall x \in \mathbb{H}^{n}$.

2. $b_{r}(x)=1$ for all $x \in B_{r / 2}(o)$.

3. $\left\|\nabla b_{r}(x)\right\| \leq \psi\left(r^{-1}\right), \forall x \in \mathbb{H}^{n}$, where $\psi(r)$ is a continuous function on $[0, \infty)$, which vanishes at 0 .

LEMma 6.6. There exists a smooth partition of unity $\left\{\eta_{i}, i \in \mathbb{N}\right\}$ subordinate to the covering $\mathcal{U}$, so that every function $\eta_{i}$ is $l_{i}$-Lipschitz, with

$$
L_{\kappa}:=\sup \left\{l_{i}: x_{i} \in M_{[\kappa, \infty)}\right\}<\infty
$$

for every $\kappa>0$.

Proof. For every $i \in I$ consider the bump-function $b_{i}$ on the ball $B_{i}$, which equals to $b_{\rho_{i}}$ after the isometric identification of $B_{i}$ with the ball $B_{\rho_{i}}(o) \subset \mathbb{H}^{n}$. Note that the radii of the balls $B_{i}$ are at least

for all

$$
\min \left(\frac{\kappa}{16}, \frac{\epsilon}{16}\right)
$$

$$
x_{i} \in M_{[\kappa, \infty)} .
$$

Therefore, for every $x_{i} \in M_{[\kappa, \infty)}$, we have

$$
\left\|\nabla b_{i}(x)\right\| \leq \lambda(\kappa)=\max \left(\psi\left(\frac{16}{\kappa}\right), \psi\left(\frac{16}{\epsilon}\right)\right)
$$

for all $x \in M$. Define the smooth partition of unity $\left\{\eta_{i}, i \in \mathbb{N}\right\}$ subordinate to the covering $\mathcal{U}$, using the bump-functions $b_{i}$ : Set

$$
c(x):=\sum_{j \in I} b_{j}(x),
$$

and

$$
\eta_{i}(x):=\frac{b_{i}(x)}{c(x)} .
$$

Then parts 1 and 4 of Proposition 6.4 imply that for every $x$,

$$
1 \leq c(x) \leq m(n, \epsilon) .
$$

Suppose that $x_{i} \in M_{[\kappa, \infty)}$ and $B_{i} \cap B_{j} \neq \emptyset$. Then, by Lemma 6.1,

$$
x_{j} \in M_{\left[\kappa^{\prime}, \infty\right)}
$$

where $\kappa^{\prime}=\frac{\kappa}{2} \cdot \frac{1-\alpha}{1+\alpha}$. In particular, if $x \in M$ is covered by the balls $B_{i_{1}}, \ldots, B_{i_{m}}$, so that $x_{i_{1}}=x_{i} \in M_{[\kappa, \infty)}$, then

$$
\|\nabla c(x)\| \leq m \lambda\left(\kappa^{\prime}\right) \leq m(n, \epsilon) \lambda\left(\kappa^{\prime}\right) .
$$

It follows that for each $x \in M$ and $x_{i} \in M_{[\kappa, \infty)}$ we have

$$
\left\|\nabla \eta_{i}(x)\right\| \leq\left|\nabla b_{i}(x)\right|+\left|b_{i}(x)\right| \cdot\|\nabla c(x)\| \leq(m(n, \epsilon)+1) \lambda\left(\kappa^{\prime}\right)=L_{\kappa} .
$$

Let $\left\{\eta_{i}, i \in I\right\}$ be a partition of unity for $M$ as above, subordinate to the covering $\mathcal{U}$ of multiplicity $m \leq m\left(n, \rho_{0}\right)$. We identify $I$ with a subset 
of $\mathbb{N}$. Let $X$ denote the $m$-dimensional simplicial complex which is the nerve of the covering $\mathcal{U}$. Collections of balls $\left\{B_{j}, j \in J\right\}$ such that

correspond to simplices $\Delta_{J} \subset X$.

$$
\bigcap_{j \in J} B_{j} \neq \emptyset
$$

We now use the above partition of unity to define a map $\eta$ from $M$ to $X$. Set

$$
\Delta^{\infty}:=\left\{z \in \mathbb{R}^{\infty}: \sum_{i=1}^{\infty} z_{i}=1, z_{i} \geq 0, i \in \mathbb{N}\right\},
$$

where

$$
\mathbb{R}^{\infty}=\bigoplus_{i \in \mathbb{N}} \mathbb{R} .
$$

Then $X$ embeds naturally in $\Delta^{\infty}$. We define the map

$$
\eta: M \rightarrow \Delta^{\infty}
$$

by

$$
\eta(x)=\left(\eta_{1}(x), \ldots, \eta_{k}(x), \ldots\right) .
$$

Since $\left\{\left(U_{i}, \eta_{i}\right)\right\}$ is a partition of unity, it is clear that the map $\eta$ is well defined. Moreover, the image of $\eta$ is contained in $X \subset \Delta^{\infty}$. Lemma 6.6 implies

Corollary 6.7. The map $\eta: M \rightarrow X$ is piecewise smooth and

is $\sqrt{m} L_{\kappa}$-Lipschitz for every $\kappa>0$.

$$
\eta \mid M_{[\kappa, \infty)}
$$

Since $\mathcal{U}$ is a covering by convex sets, the map $\eta: M \rightarrow X$ is a homotopyequivalence. Our goal is to construct its homotopy-inverse $\bar{\eta}$ with uniform control on the length of the tracks of the homotopy.

Let $\Delta_{J}=\left[e_{j_{0}}, \ldots, e_{j_{k}}\right]$ be a $k$-simplex in $X, J=\left\{j_{0}, \ldots, j_{k}\right\} \subset I$. Recall that the vertices $e_{j}, j \in J$ correspond to the balls $B_{j}=B_{\rho_{j}}\left(x_{j}\right) \in \mathcal{U}$. We define the map

$$
\bar{\eta}: \Delta_{J} \rightarrow M
$$

by sending the vertices $e_{j}$ to the corresponding centers $x_{j} \in B_{j}$. The union of the balls

$$
\bigcup_{j \in J} B_{j}
$$

is contained in a normal neighborhood $N_{x}$ in $M$ (see Corollary 6.5). Consider the convex hull

$$
\operatorname{Hull}_{J}=\operatorname{Hull}\left(\left\{x_{j}, j \in J\right\}\right) \subset N_{x} .
$$

Since $N_{x}$ is a normal neighborhood, we can regard $N_{x}$ as a subset of $\mathbb{H}^{n}$. We use the projective model for Hull $J$. Then there exists a canonical projective 
map

$$
\bar{\eta}: \Delta_{J} \rightarrow \mathrm{Hull}_{J}
$$

which extends the map defined on the vertices of $\Delta$. Namely, the projectivization $P: \mathbb{R}^{n+1} \backslash\{0\} \rightarrow \mathbb{R} P^{n}$, identifies $\mathbb{H}^{n}$ with the hyperboloid

$$
H^{n}:=\left\{\left(t_{0}, \ldots, t_{n}\right) \in \mathbb{R}^{n+1}: t_{0}>0,-t_{0}^{2}+t_{1}^{2}+\cdots+t_{n}=-1\right\} .
$$

The points $x_{j}$ are projections of the points $\hat{x}_{j} \in H^{n}$. Now, there exists a unique linear map

$$
\Delta_{J} \rightarrow \mathbb{R}^{n+1}
$$

which sends each $e_{j}$ to $\hat{x}_{j}, j \in J$. Let $\bar{\eta}$ be the composition of this map with the projection $P$.

REMARK 6.8. This is the only place in our argument where we used the fact that $M$ has constant curvature. One can avoid using the canonical projective map by appealing to convexity of the balls $B_{j}$ and defining the map $\bar{\eta}$ (noncanonically) by the induction on skeleta of $X$.

We now estimate the displacement for the composition $\bar{\eta} \circ \eta$. Set $\kappa:=$ $\epsilon / 8$.

Lemma 6.9. 1. If $x \in M$ and $z \in \operatorname{Star}(\eta(x))$, then $d(x, \bar{\eta}(z)) \leq \kappa$.

2. There exists a homotopy $H$ between $\bar{\eta} \circ \eta$ and $I d$, whose tracks have length $\leq \kappa$.

Proof. 1. Let $\Delta_{J} \subset X$ be the smallest simplex containing $\eta(x)$. If $z \in \operatorname{Star}(\eta(x))$, then $z \in \Delta_{J^{\prime}}$, where $J^{\prime} \subset I$ is a subset containing $J$, so that

$$
\bigcap_{i \in J^{\prime}} B_{i} \neq \emptyset .
$$

Let $y:=\bar{\eta}(z)$. Then

$$
y \in \bigcup_{j \in J^{\prime}} B_{j} .
$$

It follows that

$$
d(x, y) \leq 2\left(\rho_{i}+\rho_{j}\right)
$$

for some $j \in J, i \in J^{\prime}$. Since $\rho_{k} \leq \epsilon / 16$ for all $k \in I$, the first assertion of lemma follows.

2. Part 1 clearly implies that

$$
d(\bar{\eta} \circ \eta, I d) \leq \kappa=\epsilon / 8 .
$$

Moreover, for $x \in M$,

$$
y=\bar{\eta} \circ \eta(x) \in \bigcup_{j \in J} B_{j}
$$

where $J \subset I$ is defined as above. Therefore the points $x$ and $y$ belong to the convex hull Hull ${ }_{J} \subset N_{x}$. Therefore we can take the homotopy $H$ between 
$\bar{\eta} \circ \eta$ and $I d$ to be the geodesic homotopy along geodesics contained in the convex sets $\operatorname{Hull}_{J}, J \subset \mathbb{N}$ are such that $\Delta_{J} \subset X$. The length of the tracks of this homotopy clearly does not exceed $\kappa$.

Define the subcomplex $Y=Y_{\epsilon} \subset X$ to be the star of $\eta\left(M_{(0, \epsilon]}\right)$ in $X$. Hence we have the map of pairs

$$
\eta:\left(M, M_{(0, \epsilon]}\right) \rightarrow\left(X, Y_{\epsilon}\right) .
$$

Note that, because of the lack of convexity of the components of $M_{(0, \epsilon]}$, it is unclear if $\eta$ is a homotopy equivalence of pairs. Nevertheless, $\eta$ : $M \rightarrow X$ is a homotopy equivalence, hence it induces an isomorphism of the fundamental groups

$$
\Gamma=\pi_{1}(M) \rightarrow \pi_{1}(X) .
$$

We obtain a flat bundle $\mathbb{W}$ over $X$, associated with the $R \Gamma$-module $V$.

We will see that the map

$$
\eta_{\epsilon, *}: H_{*}\left(M, M_{(0, \epsilon]}, \mathbb{V}\right) \rightarrow H_{*}\left(X, Y_{\epsilon} ; \mathbb{W}\right)
$$

induced by $\eta$, is an approximate monomorphism, in the following sense:

Proposition 6.10. The kernel of $\eta_{\epsilon, *}$ is contained in the kernel of

$$
H_{*}\left(M, M_{(0, \epsilon]}, \mathbb{V}\right) \rightarrow H_{*}\left(M, M_{(0,2 \kappa+\epsilon]}, \mathbb{V}\right) .
$$

Proof. Let $\alpha \in C_{q}(M, \mathbb{W})$ be such that

$$
[\alpha] \in \operatorname{Ker}\left(\eta_{\epsilon, q}\right) \subset H_{q}\left(M, M_{(0, \epsilon]}, \mathbb{V}\right) .
$$

Let $\beta \in C_{q+1}(X, Y ; \mathbb{W})$ be a chain so that

$$
\eta_{\#}(\alpha)-\partial \beta \in C_{q}(Y ; \mathbb{W})
$$

Set

$$
\alpha^{\prime}:=(\bar{\eta} \circ \eta)_{\#}(\alpha) .
$$

Then

$$
\alpha^{\prime}-\partial \bar{\eta}_{\#}(\beta) \in C_{q}(\bar{\eta}(Y) ; \mathbb{V}) \text {. }
$$

Since $\bar{\eta}(Y)$ is contained in the $\kappa$-neighborhood of $M_{(0, \epsilon]}$, it follows that

Therefore

$$
\bar{\eta}(Y) \subset M_{(0, \epsilon+2 \kappa]} .
$$

$$
\alpha^{\prime} \in B_{q}\left(M, M_{(0, \epsilon+2 \kappa]} ; \mathbb{V}\right) \text {. }
$$

On the other hand, since the tracks of the homotopy $H$ have length $\leq \kappa$, it gives us a chain

so that

$$
\beta^{\prime} \in C_{q+1}\left(M, M_{(0, \epsilon+2 \kappa]} ; \mathbb{V}\right),
$$

Thus

$$
\alpha-\alpha^{\prime}-\partial \beta^{\prime} \in C_{*}\left(M_{(0, \epsilon+2 \kappa]} ; \mathbb{V}\right) \text {. }
$$

$$
\alpha \in B_{q}\left(M, M_{(0, \epsilon+2 \kappa]} ; \mathbb{V}\right) .
$$

REMARK 6.11. One can also prove that $\eta_{\epsilon, *}$ is an approximate epimorphism (see $[\mathrm{KK}]$ for the definition), but we will not need this. 


\section{Vanishing of Relative Homology Classes of Small Volume}

The main goal of this section is to prove the following (cf. [G, Th. 5.38]):

Theorem 7.1. There exists a function $\theta=\theta_{n}(\epsilon)$ with the following property. Let $0<\epsilon<\mu_{n} / 4$. Let $M$ be a complete (connected) hyperbolic $n$-manifold with the fundamental group $\Gamma$ and the thick-thin decomposition

$$
M=M_{(0, \epsilon]} \cup M_{[\epsilon, \infty)},
$$

where $\mu=\mu_{n}$ is the Margulis constant. Let $\mathbb{V} \rightarrow M$ be the flat bundle associated with a $R \Gamma$-module $V$. Then every relative homology class

$$
[\zeta] \in H_{q}\left(M, M_{(0, \epsilon]} ; \mathbb{V}\right), \quad q>0,
$$

whose (relative) volume is less than $\theta_{n}(\epsilon)$, is trivial.

Proof. Let $\eta: M \rightarrow X$ be the homotopy-equivalence from $M$ to the nerve of an appropriate cover, constructed in the previous section. Let $m$ denote the dimension of $X$. As $\eta$ defines an isomorphism

$$
\pi_{1}(M) \rightarrow \pi_{1}(X)
$$

we obtain the flat bundle $\mathbb{W}$ over $X$, associated with the $R \Gamma$-module $V$. The map $\eta$ induces an approximate isomorphism

$$
\eta_{*}: H_{q}\left(M, M_{(0, \epsilon]} ; \mathbb{V}\right) \rightarrow H_{q}\left(X, Y_{\epsilon} ; \mathbb{W}\right),
$$

where $Y:=Y_{\epsilon}=\operatorname{Star}\left(\eta\left(M_{(0, \epsilon]}\right)\right)$, see Proposition 6.10 .

In what follows we metrize $X$ so that every $k$-simplex in $X$ is isometric to the standard Euclidean $k$-simplex; we then will refer to $X$ as being piecewise Euclidean. Our strategy is to prove an analogue of the vanishing Theorem 7.1 first for $(X, Y)$, and then use Proposition 6.10 to derive the desired conclusion for $\left(M, M_{(0, \epsilon]}\right)$.

Proposition 7.2. There exists a constant $\nu=\nu_{m}$ with the following property. Let $X$ be an $m$-dimensional piecewise-Euclidean simplicial complex, $\mathbb{W} \rightarrow X$ be a flat bundle over $X$ and $Y \subset X$ be a subcomplex. Let $[\zeta] \in H_{q}(X, Y ; \mathbb{W})$ be a relative class of dimension $q \geq 1$ so that $\operatorname{Vol}([\zeta], Y)<\nu$. Then $[\zeta]=0$.

Proof. The idea is to retract $\zeta$ inductively to the $q$-dimensional skeleton of $X$ (away from $Y$ ) without increasing the volume too much. The resulting cycle $\zeta^{\prime}$ will have relative volume which is less than the volume of the Euclidean $q$-simplex, therefore $\zeta^{\prime}$ will miss a point in every $q$-simplex in $\overline{X \backslash Y}$. Then we retract $\zeta^{\prime}$ to the $q-1$-dimensional skeleton of $X$ away from $Y$, thereby proving vanishing of $[\zeta]$.

Lemma 7.3. Suppose that $\Delta \subset \overline{X \backslash Y}$ is a $k$-simplex, $k \geq 1$. There exists a constant $D=D(k)$ such that for every $i<k$ the following holds. 
Let $\tau \in C_{i}(\Delta, \mathbb{W})$. Then there exists a point $x \in \Delta$ which avoids the support of $\tau$ and a retraction $r: \Delta \backslash\{x\} \rightarrow \partial \Delta$ so that

$$
\operatorname{Vol}(r(\tau)) \leq D \cdot \operatorname{Vol}(\tau) .
$$

Proof. This lemma (called the deformation lemma) was proved in $[\mathrm{F}]$ in the case of the trivial $\mathbb{R}$-bundle $\mathbb{W}$ over $\Delta$. Since our bundle $\mathbb{W}$ is trivial over $\Delta$, the map $r$ defined in $[\mathrm{F}]$, extends to the restriction $\mathbb{W} \mid \Delta$ by the identity along the fibers. Since the volume of a chain is defined independently of the coefficients, it follows that the inequality (8) holds for the general flat bundles.

We set

$$
D:=\max \{D(i), q+1 \leq i \leq m\} .
$$

Lemma 7.4. Let $\tau \in C_{i}(X ; \mathbb{W})$ be a chain. Then there exists another chain $\tau^{\prime} \in C_{i}(X, \mathbb{W})$ so that

1. The support of $\tau^{\prime}$ away from $Y$ is contained in the $i$-skeleton of $X$.

2. $\operatorname{Vol}\left(\tau^{\prime}\right) \leq D^{m-i} \operatorname{Vol}(\tau)$.

3. If $\tau \in Z_{i}(X, Y ; \mathbb{W})$, then

$$
[\tau]=\left[\tau^{\prime}\right] \in H_{i}(X, Y ; \mathbb{W}) .
$$

Proof. We apply Lemma 8 inductively. Start with the $m$-skeleton of $X$. For each $m$-simplex $\Delta$ which is not contained in $Y$, we apply the retraction

$$
r: \Delta \backslash\{x\} \rightarrow \partial \Delta
$$

adapted to the $i$-chain $\tau \cap \Delta$ obtained from $\tau$ by excising $X \backslash \Delta$. We do nothing for the simplices which are contained in $Y$. The result is an $i$-chain $\tau_{1}$ such that, away from $Y$, the support of $\tau_{1}$ is contained in the $m$-1-skeleton of $X$. By Lemma 8,

$$
\operatorname{Vol}\left(\tau_{1}\right) \leq D \cdot \operatorname{Vol}(\tau) .
$$

We now repeat the above procedure with respect to the $m-1$-skeleton of $X$ and continue inductively $m-i$ times.

Let $v_{q}$ denote the volume of the standard Euclidean $q$-simplex. Set

$$
\nu=\nu(q):=v_{q} \cdot D^{q-m} .
$$

Let $[\zeta] \in H_{q}(X, Y ; \mathbb{W})$ be such that $\operatorname{Vol}(\zeta, Y)<\nu$. We claim that $[\zeta]=0$. Indeed, by applying Lemma 7.4 , we construct a relative cycle $\zeta^{\prime} \in Z_{q}(X, Y ; \mathbb{W})$ which is homologous to $\zeta$ and such that

$$
\operatorname{Vol}\left(\zeta^{\prime}\right) \leq D^{m-q} \operatorname{Vol}(\zeta)<\nu
$$

Therefore, the support of $\tau$ away from $Y$ is contained in the $q$-skeleton of $X$. Moreover, for every $q$-simplex $\Delta \subset \overline{X \backslash Y}$,

$$
\operatorname{Vol}\left(\zeta^{\prime} \cap \Delta\right) \leq \operatorname{Vol}\left(\zeta^{\prime}\right)<v_{q}=\operatorname{Vol}(\Delta) .
$$


Therefore $\zeta^{\prime}$ misses a point $x_{\Delta}$ in the interior of every $q$-simplex $\Delta \subset \overline{X \backslash Y}$. For every such $q$-simplex $\Delta$ we apply the retraction

$$
\rho_{\Delta}: \Delta \backslash\left\{x_{\Delta}\right\} \rightarrow \partial \Delta
$$

to the relative cycle $\zeta^{\prime}$. The result is a new relative cycle $\zeta^{\prime \prime}$ which is homologous to $\zeta^{\prime}$ and whose support away from $Y$ is contained in the $q-1$ skeleton of $X$. Since

it follows that

$$
H_{q}\left(X^{(q-1)}, Y^{(q-1)} ; \mathbb{W}\right)=0
$$

$$
[\zeta]=\left[\zeta^{\prime}\right]=\left[\zeta^{\prime \prime}\right]=0 \in H_{q}(X, Y ; \mathbb{W}) .
$$

Lastly, set $\nu_{m}:=\max \{\nu(q): 0<q \leq m\}$. Proposition 7.2 follows.

We are now ready to prove Theorem 7.1. Choose $\kappa>0$ so that

$$
\epsilon+2 \kappa<\mu_{n},
$$

e.g., take $\kappa=\epsilon$. By Proposition 6.4, there exists a covering $\mathcal{U}=\left\{B_{i}, i \in I\right\}$ of the manifold $M$ by $\rho_{i}$-balls $B_{i}$, where

$$
\frac{\epsilon}{16}=\sup _{i \in I} \rho_{i}
$$

$I \subset \mathbb{N}$. We obtain a piecewise-smooth map

$$
\eta: M \rightarrow X,
$$

to the nerve of this covering. The restriction of $\eta$ to $M_{[\epsilon, \infty)}$ is $L$-Lipschitz, where $L=\sqrt{m} L_{\epsilon}$, see Corollary 6.7. Set

$$
\theta:=\frac{\nu}{L^{q}}
$$

where $\nu=\nu_{m}$ is given by the Proposition 7.2.

Consider a cycle $\zeta \in Z_{q}\left(M, M_{(0, \epsilon]} ; \mathbb{V}\right)$. Then

$$
\eta_{\#}\left(\zeta \cap M_{(0, \epsilon]}\right) \subset Y \text {. }
$$

Therefore

Hence

$$
\operatorname{Vol}\left(\eta_{\#}(\zeta), Y\right) \leq \operatorname{Vol}\left(\eta_{\#}\left(\zeta \cap M_{[\epsilon, \infty}\right), Y\right) \leq L^{q} \operatorname{Vol}(\zeta) .
$$

$$
\operatorname{Vol}(\zeta, Y)<\theta \Rightarrow \operatorname{Vol}\left(\eta_{\#}(\zeta), Y\right)<\nu \Rightarrow\left[\eta_{\#}(\zeta)\right]=0 \in H_{q}\left(X, Y_{\epsilon} ; \mathbb{W}\right),
$$

by Proposition 7.2. Proposition 6.10 implies that

$$
[\zeta]=0 \in H_{q}\left(M, M_{(0, \epsilon+2 \kappa]} ; \mathbb{V}\right) \Rightarrow[\zeta]=0 \in H_{q}\left(M, M_{\left(0, \mu_{n}\right]} ; \mathbb{V}\right),
$$

since $\epsilon+2 \kappa<\mu_{n}$. Since the map (induced by the inclusion of pairs)

is an isomorphism, it follows that

$$
H_{q}\left(M, M_{(0, \epsilon]} ; \mathbb{V}\right) \rightarrow H_{q}\left(M, M_{\left(0, \mu_{n}\right]} ; \mathbb{V}\right)
$$

$$
[\zeta]=0 \in H_{q}\left(M, M_{(0, \epsilon]} ; \mathbb{V}\right) .
$$


Corollary 7.5. Let $M$ be a complete (connected) hyperbolic n-manifold as above. Let $\mathbb{V} \rightarrow M$ be the flat bundle associated with a $R \Gamma$-module $V$. Then every relative homology class

$$
[\zeta] \in H_{q}\left(M, M_{(0, \epsilon]} ; \mathbb{V}\right), \quad q>0,
$$

of zero relative volume, is trivial.

Corollary 7.6. Let $\zeta \in Z_{p}^{l f}(M ; \mathbb{V})$ be such that $\operatorname{Vol}\left(\operatorname{Exc}_{\epsilon}(\zeta)\right)<\theta(n, \epsilon)$ for some $0<\epsilon \leq \mu_{n} / 4$. Then $\zeta$ projects to 0 in $H_{p}^{\text {cusp }}(M ; \mathbb{V})$.

\section{Proof of Theorem 1.1}

Let $\Gamma \subset \operatorname{Isom}\left(\mathbb{H}^{n}\right)$ be a Kleinian group and $\Pi \subset \Gamma$ a collection of parabolic subgroups as in the Introduction. Without loss of generality, we may assume that $\Gamma$ is torsion free. Then the quotient $M=\mathbb{H}^{n} / \Gamma$ is a hyperbolic manifold. Set $\delta:=\delta(\Gamma)$. Consider an arbitrary $R \Gamma$-module $V$ and the relative homology group

$$
H_{q}(\Gamma, \Pi ; V),
$$

for $q>\delta+1$. Note that $q \geq 2$. Set

$$
\lambda:=\left(\frac{\delta+1}{q}\right)^{q} .
$$

Since $q>\delta+1$, it follows that $\lambda<1$.

As explained in section 2 , we can use the manifold $M=\mathbb{H}^{n} / \Gamma$ in order to compute the relative homology of $\Gamma$. Let $0<\epsilon<\mu_{n} / 4$, where $\mu_{n}$ is the Margulis constant for $\mathbb{H}^{n}$. Consider the $\epsilon$-thick-thin decomposition of the manifold $M$ :

$$
M=M_{(0, \epsilon]} \cup M_{[\epsilon, \infty)}
$$

and let $K:=M_{(0, \epsilon]}$. Let $P$ denote the union of components of $K$ whose fundamental group is virtually abelian of rank $\geq 2$. Then (see section 2)

$$
H_{*}(M, P ; \mathbb{V}) \cong H_{*}(\Gamma, \Pi ; V) \text {. }
$$

Lemma 8.1. Suppose that $d \geq 2$ is such that $H_{q}(M, K ; \mathbb{V})=0$ for all $q \geq d$. Then $H_{q}(M, P ; \mathbb{V})=0$ for all $q \geq d$.

Proof. Set $Q:=K \backslash P$. Then every component of $Q$ has cyclic fundamental group. Since $\mathrm{hd}_{R}(\mathbb{Z})=1$ for every ring $R$, it follows that

$$
H_{i}(Q ; \mathbb{V})=0, \quad i \geq 2 .
$$

Therefore for every $q \geq 2$ we have the following map of exact sequences:

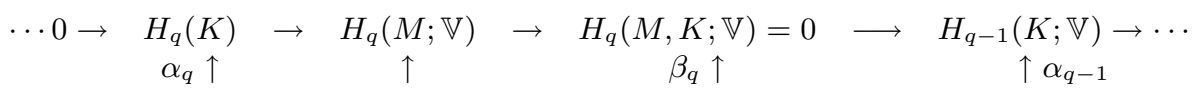

$$
\begin{aligned}
& \cdots \rightarrow H_{q}(P) \quad \rightarrow \quad H_{q}(M ; \mathbb{V}) \quad \rightarrow \quad H_{q}(M, P ; \mathbb{V}) \quad \stackrel{\delta_{q}}{\longrightarrow} \quad H_{q-1}(P ; \mathbb{V}) \rightarrow \cdots
\end{aligned}
$$


The maps $\alpha_{q}$ are isomorphisms for $q \geq 2$ and are injective for all $q$. Thus

$$
H_{q}(M, P ; \mathbb{V}) \cong H_{q}(M, K ; \mathbb{V})
$$

for $q \geq 3$. Therefore $H_{q}(M, P ; \mathbb{V})=0$ for all $q \geq \max (3, d)$. It remains to consider the case $q=d=2$. Since $\beta_{q}=0$ and $\alpha_{q-1}$ is injective, it follows that $\beta_{q}=0$. Hence $\delta_{q}=0$ and, therefore,

$$
H_{q}(M, P ; \mathbb{V}) \cong H_{q}(M ; \mathbb{V}) / H_{q}(P ; \mathbb{V}) \cong H_{q}(M ; \mathbb{V}) / H_{q}(K ; \mathbb{V})=0 .
$$

Consider a chain $\zeta \in C_{q}(M ; \mathbb{V})$ which projects to the relative homology class

$$
[\zeta] \in H_{q}(M, K ; \mathbb{V}) .
$$

Our goal is to show vanishing of the relative volume of $[\zeta]$ :

$$
\operatorname{Vol}[\zeta], K)=0
$$

i.e. that the projection of $\zeta$ to $Z_{q}(M, K ; \mathbb{V})$ is homologous to relative cycles of arbitrarily small volume. Then Theorem 7.1 will imply that $[\zeta]=0$, thereby establishing that

$$
0=H_{q}(M, P ; \mathbb{V}) \cong H_{q}(M, K ; \mathbb{V}) \cong H_{q}(\Gamma, \Pi ; V),
$$

for all $q>\delta+1$.

Let $Q^{\prime} \subset Q$ be the union of compact components and $P^{\prime}:=K \backslash Q^{\prime}$. We start by extending the chain $\zeta \in C_{q}(M ; \mathbb{V})$ to the tubes in $K$, to a chain $\zeta^{\prime}$, which projects to a relative homology class

$$
\left[\zeta^{\prime}\right]=[\zeta] \in H_{q}\left(M, P^{\prime} ; \mathbb{V}\right)
$$

see section 4.1. We then extend $\zeta^{\prime}$ to the cusps, to a locally finite cycle

$$
\hat{\zeta} \in Z_{q}(M ; \mathbb{V}) \text {, }
$$

so that

$$
v=\operatorname{Vol}(\hat{\zeta}) \leq \operatorname{Vol}\left(\zeta^{\prime}\right)+(q-1) \operatorname{Vol}\left(\partial \zeta^{\prime}\right) \text {. }
$$

Moreover,

$$
\left[\operatorname{Exc}_{\kappa}(\hat{\zeta})\right]=\left[\zeta^{\prime}\right]=[\zeta] \in H_{q}\left(M, P^{\prime} ; \mathbb{V}\right)
$$

for every $0<\kappa \leq \epsilon$. See section 4.2 . We will need

Theorem 8.2 (Besson, Courtois, Gallot, [BCG1,2]). There exists a smooth map $\tilde{F}: \mathbb{H}^{n} \rightarrow \mathbb{H}^{n}$ so that

$$
\begin{gathered}
\tilde{F} \circ \gamma=\gamma \circ \tilde{F}, \quad \forall \gamma \in \Gamma . \\
\left|\operatorname{Jac}_{r}(\tilde{F}(x))\right| \leq\left(\frac{\delta+1}{r}\right)^{r}, \quad \forall x \in \mathbb{H}^{n}, \quad \forall r \geq 1, \\
\tilde{F}(\operatorname{Hull}(\Lambda(\Gamma))) \subset \operatorname{Hull}(\Lambda(\Gamma)) .
\end{gathered}
$$

The map $\tilde{F}$ in this theorem is called a natural map. The $r$-Jacobian $\left|\operatorname{Jac}_{r}(\tilde{F}(x))\right|$ at $x \in \mathbb{H}^{n}$ is defined as

$$
\max \left\{\operatorname{Vol}\left(D_{x}(\tilde{F})\left(\xi_{1}\right), \ldots, D_{x}(\tilde{F})\left(\xi_{r}\right)\right)\right\}
$$


where the maximum is taken over all orthonormal $r$-frames $\left(\xi_{1}, \ldots, \xi_{r}\right)$ in $T_{x} \mathbb{H}^{n}$. Therefore the map $\tilde{F}$ projects to a smooth map $F: M \rightarrow M$ whose $r$-Jacobian again satisfies (11) and which is homotopic to the identity. Hence

$$
\operatorname{Vol}\left(F_{\#}(\zeta)\right) \leq \lambda \operatorname{Vol}(\zeta)
$$

see equation (9). Since $\lambda<1$, it follows that

$$
\lim _{k \rightarrow \infty} \operatorname{Vol}\left(F_{\#}^{k}(\zeta)\right)=0 .
$$

Observe that the inequality (11) applied to $r=1$, implies that the map $F$ is $(\delta+1)$-Lipschitz.

Let $\theta=\theta_{n}(\epsilon)>0$ be as in Theorem 7.1. Since $\lambda<1$, there exists $k \in \mathbb{N}$ such that

$$
\lambda^{k} v<\theta .
$$

Set $f:=F^{k}$. Then for every $\kappa \leq \epsilon$,

$$
\operatorname{Vol}\left(f_{\#}\left(\operatorname{Exc}_{\kappa}(\hat{\zeta})\right)\right) \leq \operatorname{Vol}\left(f_{\#}(\hat{\zeta})\right) \leq \lambda^{k} v<\theta .
$$

Choose $\kappa:=\epsilon /(1+\delta)^{k}$ and set

$$
\zeta^{\prime \prime}:=f_{\#}\left(\operatorname{Exc}_{\kappa}(\hat{\zeta})\right)
$$

\section{Proposition 8.3.}

$$
\left[\zeta^{\prime \prime}\right]=[\zeta]
$$

in $H_{q}\left(M, M_{(0, \epsilon]} ; \mathbb{V}\right)$.

Proof. 1. First, we have to check that

$$
\zeta^{\prime \prime} \in Z_{q}\left(M, M_{(0, \epsilon]} ; \mathbb{V}\right) \text {. }
$$

Since $f$ is $(\delta+1)^{k}$-Lipschitz, it sends the $\kappa$-thin part of $M$ to the $(\delta+1)^{k} \kappa^{-}$ thin part of $M$. Therefore

$$
\partial \zeta^{\prime \prime} \in C_{q}\left(M_{(0, \epsilon]} ; \mathbb{V}\right),
$$

which implies our assertion.

2. Since $f$ is homotopic to the identity and the cusps $P_{i}$ are pairwise disjoint, we see that

$$
f\left(P_{i} \cap M_{(0, \kappa]}\right) \subset P_{i}
$$

for every component $P_{i} \subset P^{\prime}$.

We define the straight-line homotopy $h_{t}: f \cong I d$ by projecting the straight-line homotopy

$$
\tilde{f}:=\tilde{F}^{k} \cong I d
$$

in $\mathbb{H}^{n}$. The equality

$$
\left[\zeta^{\prime \prime}\right]=[\zeta]
$$

would follow from 
Lemma 8.4. For every $x \in P_{i} \cap M_{(0, \kappa]}$, the geodesic

$$
h_{t}(x)=\overline{x f(x)}
$$

is contained in $P_{i}$.

Proof. Let $\Pi_{i} \subset \Gamma$ be the fundamental group of $P_{i}$, i.e. $\Pi_{i}$ is the stabilizer in $\Gamma$ of a component $\tilde{P}_{i}$ of the lift of $P_{i}$ to $\mathbb{H}^{n}$. Recall that $P_{i} \cap M_{(0, \kappa]}$ is the projection to $M$ of the union

$$
\bigcup_{\gamma \in \Pi_{i} \backslash\{1\}} K_{\kappa}(\gamma)
$$

see section 2. Since $\tilde{f}=\tilde{F}^{k}$ is $(1+\delta)^{k}$-Lipschitz and commutes with $\Gamma$, we obtain

$$
f\left(K_{\kappa}(\gamma)\right) \subset K_{(1+\delta)^{k} \kappa}(\gamma) \subset K_{\epsilon}(\gamma),
$$

for all $\gamma \in \Pi_{i} \backslash\{1\}$. Since $K_{\epsilon}(\gamma)$ is convex, for every $\tilde{x} \in K_{\kappa}(\gamma)$,

$$
\overline{\tilde{x} \tilde{f}(z)} \subset K_{\epsilon}(\gamma) \text {. }
$$

The above geodesic segment projects to the track $\overline{x f(x)}$ of the homotopy $h_{t}$ connecting $x=p(\tilde{x})$ to $f(x)$. On the other hand, $K_{\epsilon}(\gamma)$ projects to $P_{i}$. Therefore

$$
\overline{x f(x)} \subset P_{i} .
$$

This concludes the proof of Proposition 8.3.

We now can finish the proof of Theorem 1.1. Since

$$
\operatorname{Vol}\left(\zeta^{\prime \prime}\right)<\theta=\theta_{n}(\epsilon)
$$

and $0<\epsilon \leq \mu_{n} / 4$, Theorem 7.1 implies that $\left[\zeta^{\prime \prime}\right]=0$ in

$$
H_{q}\left(M, M_{(0, \epsilon]} ; \mathbb{V}\right) \text {. }
$$

By combining this with Proposition 8.3 , we obtain

$$
[\zeta]=\left[\zeta^{\prime \prime}\right]=0 \in H_{q}\left(M, M_{(0, \epsilon]} ; \mathbb{V}\right) .
$$

Therefore, for every $p>\delta+1$,

$$
0=H_{q}(M, P ; \mathbb{V}) \cong H_{q}(\Gamma, \Pi ; V) .
$$

Hence

$$
\operatorname{vhd}_{R}(\Gamma) \leq \delta(\Gamma)+1 .
$$

This concludes the proof of Theorem 1.1.

Proof of Corollary 1.2. Since $(\Gamma, \Pi)$ has finite type, it follows that

$$
\operatorname{cd}_{R}(\Gamma, \Pi)=\operatorname{hd}_{R}(\Gamma, \Pi)=\operatorname{vhd}_{R}(\Gamma, \Pi) \leq \delta(\Gamma)+1 .
$$

Proof of Corollary 1.5. Since $\Gamma$ is of type $F P_{2}$, it is finitely generated, hence $\Gamma$ is virtually torsion free by Selberg's lemma. Therefore, without loss of generality we may assume that $\Gamma$ is torsion free. Since $\delta\left(\mathbb{Z}^{2}\right)=1$, it 
follows that $\Gamma$ contains no free abelian subgroups of $\operatorname{rank} \geq 2$. Thus $\Pi=\emptyset$. By Theorem 1.1, we have the inequalities

$$
\operatorname{cd}_{R}(\Gamma) \leq 1+\operatorname{hd}_{R}(\Gamma) \leq \delta(\Gamma)+2<3 .
$$

The above inequality implies that $\operatorname{cd}_{R}(\Gamma) \leq 2$. Applying Lemma 2.3 to the group $\Gamma$, we conclude that $\Gamma$ is of type $F P$ and hence

$$
\operatorname{cd}_{R}(\Gamma)=\operatorname{hd}_{R}(\Gamma),
$$

see Lemma 2.9. Applying Theorem 1.1 again, we obtain the inequality

$$
\operatorname{cd}_{R}(\Gamma)=\operatorname{hd}_{R}(\Gamma) \leq \delta(\Gamma)+1<2 .
$$

Therefore $\operatorname{cd}_{R}(\Gamma)=1$ and hence $\Gamma$ is free by Theorem 2.5 .

REMARK 8.5. If one could replace vhd with vcd in Theorem 1.1, then one can weaken the assumption in Corollary 1.5 to finite generation of $\Gamma$.

\section{Application to Geometrically Finite Groups}

The main goal of this section is to prove Theorem 1.3 in the introduction. In what follows, let $\Gamma \subset \operatorname{Isom}\left(\mathbb{H}^{n}\right)$ be a nonelementary geometrically finite Kleinian group. Without loss of generality we may assume that $\Gamma$ is torsion free. Recall that $\Pi$ is a maximal collection of maximal parabolic subgroups of $\Gamma$ (of virtual rank $\geq 2$ ) which are pairwise nonconjugate and such that every maximal parabolic subgroup of $\Gamma$ is conjugate to one of the subgroups $\Pi_{i}$.

We enlarge $\Pi$ to the set

$$
\Pi^{\prime}=\left\{\Pi_{i}, i \in I\right\},
$$

which consists of representatives of conjugacy classes of all maximal parabolic subgroups in $\Gamma$. Note that

$$
\operatorname{hd}_{R}(\Gamma, \Pi)=\operatorname{hd}_{R}\left(\Gamma, \Pi^{\prime}\right),
$$

provided that $\operatorname{hd}_{R}(\Gamma, \Pi) \geq 2$, see the proof of Lemma 8.1.

We will need the following:

Proposition 9.1. Let $\Gamma \subset \operatorname{Isom}\left(\mathbb{H}^{n}\right)$ be a discrete subgroup and $\tilde{F}$ : $\mathbb{H}^{n} \rightarrow \mathbb{H}^{n}$ be the natural map associated with $\Gamma$. Suppose that there exists $\tilde{x} \in \mathbb{H}^{n}$ such that $\left|\operatorname{Jac}_{q}(\tilde{F}(\tilde{x}))\right|=1$. Then there exists a $q$-dimensional subspace $H \subset \mathbb{H}^{n}$ through $x$, so that $\operatorname{Hull}(\Lambda(\Gamma))$ is contained in $H$.

Proof. This proposition was proved in [BCG2, Prop. 5.1] in the case $q=$ $n-1$. It is clear from their proof however that it works for arbitrary $q$.

We will assume in what follows that $\Gamma$ is nonelementary and does not preserve any proper subspace in $\mathbb{H}^{n}$ (otherwise we pass to the smallest 
$\Gamma$-invariant subspace). Then the convex hull $\operatorname{Hull}(\Lambda(\Gamma))$ of $\Lambda(\Gamma)$ is $n$ dimensional.

Corollary 9.2. If there exists $x \in M$ such that $\left|\operatorname{Jac}_{q}(F(x))\right|=1$, then $q=n$.

The key technical result of this section is the following strengthening of Theorem 1.1:

Proposition 9.3. Suppose that $\Gamma \subset \operatorname{Isom}\left(\mathbb{H}^{n}\right)$ is a geometrically finite group such that $\delta(\Gamma)+1=\operatorname{cd}_{R}\left(\Gamma, \Pi^{\prime}\right)=q$. Then $q=n$ and $\Lambda(\Gamma)=S^{n-1}$.

Proof. Set $\delta:=\delta(\Gamma)$. Let $N=\operatorname{Hull}(\Lambda(\Gamma)) / \Gamma$ denote the convex core of $M$; then $N$ is $n$-dimensional. Let $\tilde{F}: \mathbb{H}^{n} \rightarrow \mathbb{H}^{n}$ be the natural map associated with $\Gamma$. Our goal is to find a point $\tilde{x} \in \mathbb{H}^{n}$ such that $\left|\operatorname{Jac}_{q}(\tilde{F}(\tilde{x}))\right|=1$. Once we found such a point, it will follow from Corollary 9.2 that $q=n$ and hence

$$
\delta=q-1=n-1 .
$$

Then, since $\Gamma$ is geometrically finite, it will follow that $\Lambda(\Gamma)=S^{n-1}$, see Theorem 2.2, part 2 .

Note that the projection $F: M \rightarrow M$ of the map $\tilde{F}$ satisfies

$$
F(N) \subset N \text {. }
$$

Moreover, since $\delta(\Gamma)+1=q$, the inequality (6) implies that $F$ does not increase the volume of chains in $C_{q}^{l f}(M ; \mathbb{V})$. Since $F$ is $(1+\delta)$-Lipschitz, the restriction $F \mid N$ is a proper map.

Since $N=K(\Gamma, 1)$, we can use the thick part of this manifold in order to compute the (relative) homology of $\Gamma$ : We choose $0<\epsilon<\mu_{n} / 4$ such that

$$
N_{(0, \epsilon]}=P_{\epsilon},
$$

is the disjoint union of cusps. Since $\Gamma$ is geometrically finite, it follows that $N_{[\epsilon, \infty)}$ is compact. Since $q=\operatorname{cd}\left(\Gamma, \Pi^{\prime}\right)$ and the pair $\left(\Gamma, \Pi^{\prime}\right)$ has finite type,

$$
q=\operatorname{cd}_{R}\left(\Gamma, \Pi^{\prime}\right)=\operatorname{hd}_{R}\left(\Gamma, \Pi^{\prime}\right) .
$$

REMARK 9.4. Without loss of generality we can assume that $\mathrm{hd}_{R}(\Gamma, \Pi) \geq 2$, since, otherwise, $\delta(\Gamma)=0$ and, hence, $\Gamma$ is elementary. Thus,

$$
\operatorname{hd}_{R}(\Gamma, \Pi)=\operatorname{hd}_{R}\left(\Gamma, \Pi^{\prime}\right)=\operatorname{cd}_{R}\left(\Gamma, \Pi^{\prime}\right) .
$$

Hence there exists an $R \Gamma$-module $V$ and a nonzero relative homology class

$$
[\zeta] \in H_{q}\left(N, P_{\epsilon} ; \mathbb{V}\right) \cong H_{q}\left(\Gamma, \Pi^{\prime} ; V\right) .
$$

Let

$$
\hat{\zeta}:=\operatorname{ext}(\zeta) \in Z_{q}^{l f}(N ; \mathbb{V})
$$


be a finite volume extension of the relative cycle $\zeta$. Since $\operatorname{Vol}(\hat{\zeta})<\infty$, there exists $0<\kappa<\epsilon$ such that the chain $\zeta^{\prime \prime}:=\hat{\zeta} \cap P_{\kappa}$ satisfies

$$
\operatorname{Vol}\left(\zeta^{\prime \prime}\right)<t:=\theta_{n}(\epsilon) / 2,
$$

where $\theta_{n}(\epsilon)$ is the function introduced in Theorem 7.1. Therefore

$$
\operatorname{Vol}\left(F^{k}\left(\zeta^{\prime \prime}\right)\right)<t
$$

for all $k \geq 0$. Set $\zeta^{\prime}:=\operatorname{Exc}_{\kappa}(\hat{\zeta})=\hat{\zeta} \cap N_{[\kappa, \infty)}$.

For $k \in \mathbb{N}$ define the chains

$$
\zeta_{k}^{\prime}:=F^{k}\left(\zeta^{\prime}\right), \quad \zeta_{k}^{+}:=\zeta_{k}^{\prime} \cap N_{[\epsilon, \infty)}, \quad \zeta_{k}^{-}:=\zeta_{k}^{\prime} \cap N_{(0, \epsilon]} .
$$

We will consider two cases, in the first case we find a point $y \in N$ such that $\left|\operatorname{Jac}_{q}(F(y))\right|=1$, the second case will be ruled out as it will lead to the contradiction with nonvanishing of $[\zeta]$.

Case 1. Let $\chi$ denote the characteristic function of $N_{[\epsilon, \infty)}$. Suppose that there exists a sequence $x_{j} \in \operatorname{Supp}\left(\zeta^{\prime}\right)$ and $k_{j} \in \mathbb{N}$, such that for $y_{j}:=F^{k_{j}}\left(x_{j}\right)$ we have

$$
\lim _{j} \chi\left(y_{j}\right) \cdot\left|\operatorname{Jac}_{q}\left(F\left(y_{j}\right)\right)\right|=1 \text {. }
$$

Then the sequence $\left(y_{j}\right)$ belongs to the compact $N_{[\epsilon, \infty)}$ and hence subconverges to a point $y$ so that

$$
\left|\operatorname{Jac}_{q}(F(y))\right|=1 \text {. }
$$

Thus we are done by Corollary 9.2.

Case 2. Otherwise, there exists $0<\lambda<1$ so that for all $k \in \mathbb{N}$,

$$
\operatorname{Supp}\left(\zeta_{k}^{+}\right) \subset E_{\lambda}:=\left\{x \in M:\left|\operatorname{Jac}_{q}(F(x))\right| \leq \lambda\right\} .
$$

Lemma 9.5. There exists $k \in \mathbb{N}$ such that $\operatorname{Vol}\left(\zeta_{k}^{\prime}, P_{\epsilon}\right)<t=\theta_{n}(\epsilon) / 2$.

Proof. Suppose not. Then for every $k$ we have

and hence

$$
\operatorname{Vol}\left(\zeta_{k}^{+}\right) \geq t
$$

$$
\frac{\operatorname{Vol}\left(\zeta_{k}^{+}\right)}{\operatorname{Vol}\left(\zeta_{k}^{\prime}\right)} \geq \frac{t}{v}
$$

where

$$
v:=\operatorname{Vol}\left(\zeta^{\prime}\right) \geq \operatorname{Vol}\left(\zeta_{k}^{\prime}\right)
$$

Moreover, by (13), we get

$\operatorname{Vol}\left(\zeta_{k+1}^{\prime}\right)=\operatorname{Vol}\left(F\left(\zeta_{k}^{\prime}\right)\right) \leq \lambda \operatorname{Vol}\left(\zeta_{k}^{+}\right)+\operatorname{Vol}\left(\zeta_{k}^{-}\right) \leq\left[(\lambda-1) \frac{t}{v}+1\right] \operatorname{Vol}\left(\zeta_{k}^{\prime}\right)$.

Note that, since $\lambda<1$, we have

Therefore

$$
0<\left[(\lambda-1) \frac{t}{v}+1\right]<1 .
$$

$$
\lim _{k \rightarrow \infty} \operatorname{Vol}\left(\zeta_{k}^{\prime}, P_{\epsilon}\right) \leq \lim _{k \rightarrow \infty} \operatorname{Vol}\left(\zeta_{k}^{\prime}\right)=0
$$


This contradicts the assumption that $\operatorname{Vol}\left(\zeta_{k}^{\prime}, P_{\epsilon}\right) \geq t>0$ for all $k$. Contradiction.

We now can finish the proof of the proposition. We first estimate $\operatorname{Vol}\left(F^{k}(\hat{\zeta}), P_{\epsilon}\right)$ for the number $k$ guaranteed by the above lemma:

$\operatorname{Vol}\left(F^{k}(\hat{\zeta}), P_{\epsilon}\right) \leq \operatorname{Vol}\left(F^{k}\left(\zeta^{\prime}\right), P_{\epsilon}\right)+\operatorname{Vol}\left(F^{k}\left(\zeta^{\prime \prime}\right)\right) \leq t+t=\theta_{n}(\epsilon)$, since $\operatorname{Vol}\left(F^{k}\left(\zeta^{\prime \prime}\right)\right) \leq t$.

Hence, by Corollary 7.6 , the locally finite cycle $F^{k}(\hat{\zeta})$ projects to zero class

$$
\left[\operatorname{Exc}_{\epsilon}\left(F^{k}(\hat{\zeta})\right)\right] \in H_{q}^{c u s p}(M ; \mathbb{V}) .
$$

Since $F^{k}: N \rightarrow N$ is Lipschitz and commutes with $\Gamma$, it is properly homotopic to the identity. Therefore

Thus

$$
[\hat{\zeta}]=F^{k}([\hat{\zeta}]) \in H_{q}^{l f}(M ; \mathbb{V}) .
$$

$$
[\zeta]=\left[\operatorname{Exc}_{\epsilon}(\hat{\zeta})\right]=0 \in H_{q}\left(M, P_{\epsilon} ; \mathbb{V}\right) .
$$

This contradicts the assumption that $[\zeta]$ is a nonzero class in $H_{q}\left(M, P_{\epsilon} ; \mathbb{V}\right)$.

In order to relate the above proposition to the limit set of $\Gamma$ we will need the following proposition, which is a relative version of a theorem by Bestvina and Mess in $[\mathrm{BeM}]$ :

Proposition 9.6. $\operatorname{cd}\left(\Gamma, \Pi^{\prime}\right)=\operatorname{dim}(\Lambda(\Gamma))+1$, where dim is the topological dimension.

Proof. Without loss of generality we may assume that $\Gamma$ is torsion free. Let $H:=\operatorname{Hull}(\Lambda)$ denote the convex hull of the limit set $\Lambda$ of the group $\Gamma$. The set $H$ is obviously contractible. Moreover, the union

$$
H \cup \Lambda
$$

satisfies the axioms of the $\mathcal{Z}$-set compactification of $H$ and therefore

$$
H_{c}^{*}(H) \cong \check{H}^{*-1}(\Lambda) \text {, }
$$

see $[\mathrm{BeM}]$ and $[\mathrm{Be}]$. Since $\Gamma$ is geometrically finite, the pair $\left(\Gamma, \Pi^{\prime}\right)$ has finite type; hence

see Lemma 2.9 .

$$
\operatorname{cd}\left(\Gamma, \Pi^{\prime}\right)=\sup \left\{q: H^{q}\left(\Gamma, \Pi^{\prime} ; \mathbb{Z} \Gamma\right) \neq 0\right\},
$$

Since $\Gamma$ is geometrically finite, for every $i \in I$ we can choose a closed horoball $B_{i}$, centered at the fixed point of $\Pi_{i}$, so that

$$
\gamma\left(B_{i}\right) \cap B_{j}=\emptyset
$$

unless $i=j$ and $\gamma \in \Pi_{i}$, in which case $\gamma\left(B_{i}\right)=B_{j}$. See for instance [Bow].

Therefore the set

$$
H^{\prime}:=H \backslash \Gamma \cdot \bigcup_{i \in I} \operatorname{int}\left(B_{i}\right)
$$

projects to a compact submanifold with boundary $N^{\prime}$ in $N=H / \Gamma$. 
For every $i \in I$, set

$$
\begin{aligned}
& C_{i}:=B_{i} \cap H, \\
& C:=\Gamma \cdot \bigcup_{i \in I} C_{i},
\end{aligned}
$$

and

$$
\begin{aligned}
C_{i}^{\prime} & :=B_{i} \cap H^{\prime}, \\
C^{\prime} & :=\Gamma \cdot \bigcup_{i \in I} C_{i}^{\prime} .
\end{aligned}
$$

Then convexity of $H$ and of every $B_{i}$ implies that $H^{\prime}$ and each $C_{i}^{\prime}$ is contractible. Therefore $N^{\prime}=H^{\prime} / \Gamma$ is a compact $K(\Gamma, 1)$ and $C_{i}^{\prime} / \Pi_{i}$ is a compact $K\left(\Pi_{i}, 1\right)$, for every $i \in I$.

Lemma 9.7. $\quad H_{c}^{*}(C)=0$.

Proof. Since each $B_{i}$ is a horoball centered at a limit point of $\Gamma$ and $H$ is convex, it follows that

$$
C \cong[0,1) \times C^{\prime} .
$$

Therefore vanishing of $H_{c}^{*}([0,1))$ implies vanishing of $H_{c}^{*}(C)$.

Hence, by the long exact sequence of the pair $(H, C)$, we have

$$
H_{c}^{*}(H) \cong H_{c}^{*}(H, C) \text {. }
$$

We claim that

$$
H^{*}\left(\Gamma, \Pi^{\prime} ; \mathbb{Z} \Gamma\right) \cong H_{c}^{*}\left(H^{\prime}, C^{\prime}\right) \cong H_{c}^{*}(H, C) \cong H_{c}^{*}(H) \cong \check{H}^{*-1}(\Lambda) .
$$

The first isomorphism in this sequence is established in [Bro, Ch. VIII, Prop. 7.5] in the case $\Pi=\emptyset$ and $\Gamma$ of finite type with $H^{\prime}$ being the universal cover of a compact $K(\Gamma, 1)$; the general case follows from the long exact sequences of the pairs $(\Gamma, \Pi),\left(H^{\prime}, C^{\prime}\right)$. The rest of the isomorphisms were established above. Hence $\operatorname{cd}\left(\Gamma, \Pi^{\prime}\right)=\operatorname{dim}(\Lambda(\Gamma))+1$ and Proposition 9.6 follows.

REMARK 9.8. The proof of Proposition 9.6 generalizes without much difficulty to the case when $\Gamma$ is a relatively hyperbolic group with respect to a family $\Pi^{\prime}$ of virtually nilpotent subgroups. The limit set in this case is replaced by the Bowditch boundary of $\Gamma$.

We are now ready to prove Theorem 1.3. By the assumption,

$$
\operatorname{dim}_{H} \Lambda(\Gamma)=\operatorname{dim} \Lambda(\Gamma) .
$$

Since, by Theorem 2.2, for nonelementary geometrically finite Kleinian groups $\Gamma$ we have

$$
\operatorname{dim}_{H} \Lambda(\Gamma)=\delta(\Gamma),
$$


Proposition 9.6 implies

$$
\delta(\Gamma)=\operatorname{dim}_{H} \Lambda(\Gamma)=\operatorname{dim} \Lambda(\Gamma)=\operatorname{cd}\left(\Gamma, \Pi^{\prime}\right)-1 .
$$

Lastly, Proposition 9.3 implies that $\Gamma$ is a lattice.

\section{References}

[A] I. AGOL, Tameness of hyperbolic 3-manifolds, preprint (2004); math. GT/0405568

[BCG1] G. Besson, G. Courtois, S. Gallot, Hyperbolic manifolds, amalgamated products and critical exponents, C.R. Math. Acad. Sci. Paris, 336 (2003), 257-261.

[BCG2] G. Besson, G. Courtois, S. Gallot, Rigidity of amalgamated product in negative curvature, Journ. Diff. Geom. 79 (2008), 335-387.

[Be] M. Bestvina, Local homology properties of boundaries of groups, Michigan Math. J. 43 (1996), 123-139.

[BeB] M. Bestvina, N. Brady, Morse theory and finiteness properties of groups, Inventiones Math. 129 (1997), 445-470.

[BeM] M. Bestvina, G. Mess, The boundary of negatively curved groups, Journal of the AMS 4 (1991), 469-481.

[Bi] R. BIERI, Homological Dimension of Discrete Groups, Mathematical Notes, Queen Mary College, 1976.

[BiE] R. Bieri, B. Eckmann, Relative homology and Poincaré duality for group pairs, J. Pure Appl. Algebra 13 (1978), 277-319.

[BisJ] C. Bishop, P. Jones, Hausdorff dimension and Kleinian groups, Acta Math. 179 (1997), 1-39.

[BoK] M. BonK, B. KLEINER, Rigidity for quasi-Fuchsian actions on negatively curved spaces, Int. Math. Res. Not. (2004), 3309-3316.

[Bow] B.H. BowDitch, Geometrical finiteness for hyperbolic groups, Journal of Functional Anal. 113 (1993), 245-317.

[Bowe] R. Bowen, Hausdorff dimension of quasi-circles, Math. Publ. IHES (1979), 11-25.

[Br] W. Breslin, Curvatures of Surfaces in Hyperbolic 3-Manifolds, PhD Thesis, UC Davis, 2007.

[Bro] K. Brown, Cohomology of Groups Graduate Texts in Mathematics 87, Springer (1982).

[CG] D. Calegari, D. Gabai, Shrinkwrapping and the taming of hyperbolic 3-manifolds, J. Amer. Math. Soc. 19 (2006), 385-446.

[D] M.J. Dunwoody, Accessibility and groups of cohomological dimension one, Proc. London Math. Soc. (3) 38 (1979), 193-215.

[F] H. Federer, Geometric measure theory, Die Grundlehren der mathematischen Wissenschaften, Band 153, Springer-Verlag New York Inc., New York (1969). 
[G] M. Gromov, Metric Structures for Riemannian and Non-Riemannian Spaces, Birkhäuser Boston, 1999.

$[\mathrm{H}] \quad$ Y. Hou, Kleinian groups of small Hausdorff dimension are classical Schottky groups, preprint (2006).

[HuW] W. Hurewicz, H. Wallman, Dimension Theory, Princeton University Press, 1941.

[JM] D. Johnson, J.J. Millson, Deformation spaces associated to compact hyperbolic manifolds, in "Discrete Groups in Geometry and Analysis", Papers in honor of G.D. Mostow on his 60-th birthday, (R. Howe, ed.), Progress in Mathematics, Birkhauser (1987), 48-106.

[KK] M. Kapovich, B. KLeiner, Coarse Alexander duality and duality groups, Journal of Differential Geometry 69 (2005), 279-352.

[KP] M. Kapovich, L. Potyagailo, On absence of Ahlfors' and Sullivan's finiteness theorems for Kleinian groups in higher dimensions, Siberian Math. Journ. 32 (1991), 227-237.

[N] P.J. Nicholls, The Ergodic Theory of Discrete Groups, Cambridge University Press, 1989.

[S] J. Stallings, On torsion-free groups with infinitely many ends, Ann. of Math. 88 (1968), 312-334.

[Su] D. Sullivan, Entropy, Hausdorff measures old and new, and limit sets of geometrically finite Kleinian groups, Acta. Math. 153 (1984), 259-277.

[Sw] R. Swan, Groups of cohomological dimension one, Journal of Algebra 12 (1969), 585-601.

[T1] W. Thurston, The Geometry and Topology of 3-manifolds, Princeton University Lecture Notes, 1978.

[T2] W. Thurston, Hyperbolic structures on 3-manifolds, I, Ann. of Math. 124 (1986), 203-246.

[Tu] P. Tukia, The Hausdorff dimension of the limit set of a geometrically finite Kleinian group, Acta Math. 152 (1984), 127-140.

[X] X. XIE, A Bowen type rigidity theorem for non-compact hyperbolic groups, Mathematische Zeitschrift 259 (2008), 249-253.

[Y] C. Yue, Dimension and rigidity of quasifuchsian representations, Ann. of Math. 143 (1996), 331-355.

Michael Kapovich, Department of Mathematics, University of California, Davis, CA 95616, USA

kapovich@math.ucdavis.edu

Received: March 2007

Revision: October 2007

Accepted: October 2007 